\title{
On the Commutative Rings with At Most Two Proper Subrings
}

\author{
David E. Dobbs \\ Department of Mathematics, University of Tennessee, Knoxville, TN 37996-1320, USA \\ Correspondence should be addressed to David E. Dobbs; dobbs@math.utk.edu \\ Received 4 April 2016; Accepted 6 June 2016 \\ Academic Editor: Kaiming Zhao
}

Copyright ( 2016 David E. Dobbs. This is an open access article distributed under the Creative Commons Attribution License, which permits unrestricted use, distribution, and reproduction in any medium, provided the original work is properly cited.

The commutative rings with exactly two proper (unital) subrings are characterized. An initial step involves the description of the commutative rings having only one proper subring.

\section{Introduction}

This paper is a sequel to [1]. All rings considered below are commutative with identity; all subrings, inclusions of rings, ring extensions, ring or algebra homomorphisms, modules, and submodules are unital. Our interest here is in characterizing rings that have at most two proper subrings. Observe that a ring $R$ has no proper subrings if and only if either $R=0, R \cong \mathbb{Z}$, or $R \cong \mathbb{Z} / n \mathbb{Z}$ for some integer $n \geq 2$ and that these three cases are mutually exclusive. We will answer the analogous questions for rings having exactly one proper subring (resp., for rings having exactly two proper subrings) in Theorems 5 and 13 (resp., in Theorems 6 and 15). In doing so, we may tacitly ignore the zero ring, as $R=0$ does not have any proper subrings (or, for that matter, any proper ring extensions). Thus, we may organize our answers with a focus on the characteristic of a ring of interest, since every nonzero ring is a ring extension of an isomorphic copy of exactly one of the so-called prime rings (namely, $\mathbb{Z}$ and $\mathbb{Z} / n \mathbb{Z}$ for $n \geq 2$ ).

Notice that a ring $R$ has exactly one proper subring if and only if $R$ is a minimal ring extension (in the sense of [2]) of its prime (sub)ring. As the minimal ring extensions of a (commutative integral) domain were classified in [3], much of the technical work leading up to our main theorems will be concerned with determining the minimal ring extensions of the prime rings of positive characteristic. Then, by determining the minimal ring extensions of either those minimal ring extensions or the minimal ring extensions of $\mathbb{Z}$, we will be led to a list of candidates for the characterizations in Theorems 6 and 15. Determining which of those candidates survive will depend on the main result of [1] which, for convenience, is restated here as Theorem 2. Carrying out this program will require details about minimal ring extensions, most of which are summarized in the next paragraph.

Recall (cf. [2]) that a ring extension $A \subset B$ is a minimal ring extension if there does not exist a ring properly contained between $A$ and $B$. A minimal ring extension $A \subset B$ is either integrally closed (in the sense that $A$ is integrally closed in $B$ ) or integral. If $A \subset B$ is a minimal ring extension, it follows from [2, Théorème 2.2(i) and Lemme 1.3] that there exists a unique maximal ideal $M$ of $A$ (called the crucial maximal ideal of $A \subset B$ ) such that the canonical injective ring homomorphism $A_{M} \rightarrow B_{M}\left(:=B_{A \backslash M}\right)$ can be viewed as a minimal ring extension while the canonical ring homomorphism $A_{P} \rightarrow B_{P}$ is an isomorphism for all prime ideals $P$ of $A$ except $M$. A minimal ring extension $A \subset B$ is integrally closed if and only if $A \hookrightarrow B$ is a flat epimorphism (in the category of commutative rings). If $A \subset B$ is an integral minimal ring extension with crucial maximal ideal $M$, there are three possibilities: $A \subset B$ is said to be, respectively, inert, ramified, or decomposed if $B / M B(=B / M)$ is isomorphic, as an algebra over the field $K:=A / M$, to a minimal field extension of $K, K[X] /\left(X^{2}\right)$, or $K \times K$. (As usual, $X$ denote an indeterminate over the ambient base ring.)

If $A$ is a ring, then $\operatorname{char}(A)$ denotes the characteristic of $A ; \operatorname{Spec}(A)$ (resp., $\operatorname{Max}(A))$ denotes the set of prime (resp., maximal) ideals of $A$; and by the dimension of $A$, we mean the Krull dimension of $A$, denoted by $\operatorname{dim}(A)$. If $A \subseteq B$ are rings, then $[A, B]$ denotes the set of rings $C$ such that $A \subseteq C \subseteq B$; and, as in [4], we say that $A \subseteq B$ satisfies FIP (for the "finitely many intermediate rings" property) if the set $[A, B]$ is finite. Following [5, page 28], we let INC, LO, and GU, respectively, 
denote the incomparable, lying-over, and going-up properties of ring extensions. Given rings $A \subseteq B$ with $P \in \operatorname{Spec}(A)$, then $B_{P}:=B_{A \backslash P}$. As usual, $\subset$ denotes proper inclusion; $\mathbb{F}_{q}$ denotes the finite field of cardinality $q$; and $|H|$ denotes the cardinality of a set $H$. The reader may find it useful to have copies of $[1,6]$ at hand. Any otherwise unexplained material is standard, as in [5].

\section{Results}

Our work will be separated into individual approaches that depend on the characteristic of the ambient prime ring. Each of those approaches will make use of the next two results, which are the main results of $[1,6]$. Prior to giving the main results for each of our approaches, we will provide most of the new technical results that will be needed to prove those main results. We will not need to make the cumbersome conditions from [6, Proposition 3.5] (ultimately, from [7, Theorem 5.18]) explicit which are mentioned in parts (xii) and (xiii) of Theorem 1.

Theorem 1 (see [6, Theorem 4.1]). Let $R \subset S$ and $S \subset T$ be minimal ring extensions, with crucial maximal ideals $M$ and $N$, respectively. Then $R \subset T$ satisfies FIP if and only if (exactly) one of the following conditions holds:

(i) Both $R \subset S$ and $S \subset T$ are integrally closed.

(ii) $R \subset S$ is integral and $S \subset T$ is integrally closed.

(iii) $R \subset S$ is integrally closed, $S \subset T$ is integral, and $N \cap R \nsubseteq$ $M$.

(iv) Both $R \subset S$ and $S \subset T$ are integral and $N \cap R \neq M$.

(v) Both $R \subset S$ and $S \subset T$ are inert, $N \cap R=M$, and either $R / M$ is finite or there exists $\gamma \in T_{M}$ such that $T_{M}=R_{M}[\gamma]$.

(vi) $R \subset S$ is decomposed, $S \subset T$ is inert, and $N \cap R=M$.

(vii) Both $R \subset S$ and $S \subset T$ are decomposed and $N \cap R=M$.

(viii) $R \subset S$ is inert, $S \subset T$ is decomposed, $N \cap R=M$.

(ix) $R \subset S$ is ramified, $S \subset T$ is decomposed, and $N \cap R=$ $M$.

(x) $R \subset S$ is decomposed, $S \subset T$ is ramified, and $N \cap R=$ $M$.

(xi) $R \subset S$ is ramified, $S \subset T$ is inert, and $N \cap R=M$.

(xii) $R \subset S$ is inert, $S \subset T$ is ramified, $N \cap R=M$, and the two conditions stated in [6, Proposition 3.5(a)] hold.

(xiii) Both $R \subset S$ and $S \subset T$ are ramified, $N \cap R=M$, and the two conditions stated in [6, Proposition 3.5(b)] hold.

Theorem 2 (see [1, Theorem 2.9]). Consider the 13 conditions, (i)-(xiii), in the statement of Theorem 1. Then, consider the following:

(a) If data satisfy condition (vi) or condition (xi), then $|[R, T]|=3$.

(b) If data satisfy any of the seven conditions (iii), (iv), (vii), (viii), (ix), (x), and (xii), then $|[R, T]|>3$ (and $|[R, T]|<\infty)$. (c) For each of the four conditions (i), (ii), (v), and (xiii), there exist data satisfying this condition for which $|[R, T]|=3$ and there exist other data satisfying this condition for which $|[R, T]|>3$ (and $|[R, T]|<\infty$ ).

We will often use the fact [8, Theorem 25.1(3)] that if $R$ is a ring and $E$ is an $R$-module, then $\operatorname{Spec}(R(+) E)=\{P(+) E \mid$ $P \in \operatorname{Spec}(R)\}$. As we now begin the approach that is specific to the context of characteristic 0 , it is also convenient to record here another fact that will see frequent use below. To wit: the integrally closed minimal ring extensions of $\mathbb{Z}$ are, up to isomorphism, the minimal overrings of $\mathbb{Z}$ (inside $\mathbb{Q}$ ), namely, the rings $\mathbb{Z}[1 / s]$, where $s$ runs over the set of prime numbers. This fact seems to be well known and can be recovered from [9, Lemma V.2] (cf. also [3, Remark 2.8(a)]). More generally, the minimal overrings of an arbitrary principal ideal domain have been explicitly identified earlier: see, for instance, [10, Proposition 4.11].

Proposition 3. Let $p$ be a prime number and $A:=\mathbb{Z}(+) \mathbb{F}_{p}$. Then, consider the following:

(a) Let $q$ be a prime number that is distinct from $p$. Then, up to A-algebra isomorphism, $B:=\mathbb{Z}[1 / q](+) \mathbb{F}_{p}$ is the unique ring such that $A \subset B$ is an integrally closed minimal ring extension whose crucial maximal ideal is $q \mathbb{Z}(+) \mathbb{F}_{p}$.

(b) There does not exist a ring $S$ such that $A \subset S$ is an integrally closed minimal ring extension whose crucial maximal ideal is $p \mathbb{Z}(+) \mathbb{F}_{p}$.

Proof. We will show first that if $q$ is a prime number that is distinct from $p$ and we put $T:=\mathbb{Z}[1 / q](+) \mathbb{F}_{p}$, then $A \subset T$ is an integrally closed minimal ring extension whose crucial maximal ideal is $q \mathbb{Z}(+) \mathbb{F}_{p}$. The "minimal ring extension" assertion follows from the above comments because each member of $[A, T]$ takes the form $D(+) \mathbb{F}_{p}$ for some (uniquely determined) $D \in[\mathbb{Z}, \mathbb{Z}[1 / q]]$. The remaining parts of the assertion follow via the lore of the idealization construction. Indeed, by [8, Theorem 25.5(1)], the total quotient ring of $A$ is (canonically isomorphic to) $\mathbb{Z}_{p \mathbb{Z}}(+)\left(\mathbb{F}_{p}\right)_{\mathbb{Z} \backslash p \mathbb{Z}}$, that is, to $\mathbb{Z}_{p \mathbb{Z}}(+) \mathbb{F}_{p}$. As $\mathbb{Z}$ is integrally closed (in $\mathbb{Q}$ and, hence, in $\mathbb{Z}_{p \mathbb{Z}}$ ), it now follows from $[8$, Theorem 25.5(1)] that $A$ coincides with its integral closure (in its total quotient ring). In particular, $A \subset T$ is an integrally closed ring extension. To show that the crucial maximal ideal of this extension is $I:=q \mathbb{Z}(+) \mathbb{F}_{p},[2$, Théorème 2.2(i)] reduces our task to showing that $A_{I} \subset T_{I}$. Using [8, Corollary 25.5(2)], we find canonical identifications

$$
A_{I}=\mathbb{Z}_{q \mathbb{Z}}(+)\left(\mathbb{F}_{p}\right)_{\mathbb{Z} \backslash q \mathbb{Z}}=\mathbb{Z}_{q \mathbb{Z}}(+) 0=\mathbb{Z}_{q \mathbb{Z}}
$$

and, similarly, $T_{I}=(\mathbb{Z}[1 / q])_{\mathbb{Z} \backslash q \mathbb{Z}}(+) 0=\mathbb{Q}$.

It now suffices to show that if a ring $B$ is such that $A \subset$ $B$ is an integrally closed minimal ring extension, then up to $A$-algebra isomorphism, $B=\mathbb{Z}[1 / q](+) \mathbb{F}_{p}$ for some prime number $q \neq p$. By [2, Théorème 2.2(iii)], the inclusion map $A \hookrightarrow B$ is a flat epimorphism (in the category of rings). We claim that, up to $A$-algebra isomorphism, $B$ is an overring of $A$ (inside the total quotient ring of $A$ ); that is, we claim that we can view $B \subseteq \mathbb{Z}_{p \mathbb{Z}}(+) \mathbb{F}_{p}$. 
Since $A \hookrightarrow B$ is a flat epimorphism, we can view $B \subseteq$ $M(A)$, where $M(A)$ is a certain universal object introduced by Lazard in [11]. Hence, to prove the above claim, it suffices to prove that $M(A)$ coincides with the total quotient ring of $A$. Therefore, by the proof of [11, Proposition 4.1, page $116]$, it suffices to show that the generization (inside $\operatorname{Spec}(A)$ ) of the set of weak Bourbaki associated primes of $A$ is the same as the canonical image inside $\operatorname{Spec}(A)$ of the set of all prime ideals of the total quotient ring of $A$, that is, the same as the two-element set whose members are $P_{1}:=p \mathbb{Z}(+) \mathbb{F}_{p}$ and $P_{2}:=0(+) \mathbb{F}_{p}$. (Some authors translate "le générisé de" as "the generalization of"; we prefer the translation "the generization of " because it is more reminiscent of the relevant notion of a generic point.) By definition, a weak Bourbaki associated prime of $A$ is a minimal prime (ideal of $A$ ) over the annihilator of some element of $A$. A routine case analysis shows that the set of such annihilators consists of $p \mathbb{Z} \times\{0\},\{0\} \times \mathbb{F}_{p}$, and $\{(0,0)\}$. It follows that the set of weak Bourbaki associated primes of $A$ is $\left\{P_{1}, P_{2}\right\}$. As this set is stable under generization, the above claim has been proved.

We have shown that $A \subset B \subseteq \mathbb{Z}_{p \mathbb{Z}}(+) \mathbb{F}_{p}$. It follows easily that $B=E(+) \mathbb{F}_{p}$ for some ring $E$ such that $E \subseteq \mathbb{Z}_{p \mathbb{Z}}$ and $\mathbb{Z} \subset$ $E$ is a minimal ring extension. By the above comments, $E=$ $\mathbb{Z}[1 / q]$ for some prime number $q$. In short, $B=\mathbb{Z}[1 / q](+) \mathbb{F}_{p}$. Finally, $q \neq p$ since $1 / q \in E \subseteq \mathbb{Z}_{p \mathbb{Z}}$.

Proposition 4. Let $p$ be a prime number and $A:=\mathbb{Z}(+) \mathbb{F}_{p}$. Then there does not exist a ring $B$ such that $A \subset B$ is an inert extension whose crucial maximal ideal is $p \mathbb{Z}(+) \mathbb{F}_{p}$.

Proof. Put $N:=p \mathbb{Z}(+) \mathbb{F}_{p}(=p \mathbb{Z}(+) \mathbb{Z} / p \mathbb{Z})$. Then we can identify $A_{N}=\mathbb{Z}_{p \mathbb{Z}}(+) \mathbb{Z} / p \mathbb{Z}$ (cf. [8, Lemma 25.4 and Theorem 25.5(2)]). Now, suppose the assertion fails. Then $N \in \operatorname{Max}(B)$; and by [12, Proposition 4.6], $A_{N} \subset B_{N}(:=$ $\left.B_{A \backslash N}\right)$ is an inert extension. In particular, $B_{N} / N B_{N} \cong B / N$ is a minimal field extension of $A / N\left(\cong \mathbb{F}_{p}\right) \cong A_{N} / N A_{N}$. Hence, by a harmless change of notation, we can now take $A:=\mathbb{Z}_{p \mathbb{Z}}(+) \mathbb{Z} / p \mathbb{Z}$, with $A \subset B$ being an inert extension, necessarily with crucial maximal ideal $N=p \mathbb{Z}(+) \mathbb{Z} / p \mathbb{Z}$. As we can view $\mathbb{F}_{p} \rightarrow B / N$ as a minimal field extension, the standard Galois theory of finite fields provides a prime number $q$ (possibly equal to $p$ ) such that $B / N=\mathbb{F}_{p^{q}}$, whence $y^{p^{q}}=y$ for each element $y \in B / N$.

Pick $e \in B \backslash A$. Then $A[e]=B$ by the minimality of $A \subset B$. Also, since the integral extension $A \subset B$ must satisfy INC, LO, and GU (cf. [5, Theorem 44]), we see that $(B, N)$ is quasi-local and, in fact, that $\operatorname{Spec}(A)=\operatorname{Spec}(B)$ as sets. Since $x:=(p, 1+$ $p \mathbb{Z}) \in N$ is a (nonzero) non-zero-divisor in $A$, it follows that $x$ is a non-zero-divisor in $B$. Also, since $x e \in N B=N$, there exist $z, a \in \mathbb{Z}$ such that $x e=(p z, a+p \mathbb{Z})$. Thus, $b:=a-z \in \mathbb{Z}$ satisfies

$$
\begin{aligned}
x(e-(z, 0)) & =x e-x(z, 0) \\
& =(p z, a+p \mathbb{Z})-(p z, z+p \mathbb{Z}) \\
& =(0, b+p \mathbb{Z}) .
\end{aligned}
$$

Taking $p^{q}$ th powers, we get

$$
\begin{aligned}
x^{p^{q}}(e-(z, 0))^{p^{q}} & =(x(e-(z, 0)))^{p^{q}}=(0, b+p \mathbb{Z})^{p^{q}} \\
& =(0,0) .
\end{aligned}
$$

As $x^{p^{q}}$ is a non-zero-divisor in $B$, we now have $(e-(z, 0))^{p^{q}}=$ $(0,0) \in A \subset B$. Applying the canonical surjection $B \rightarrow B / N$ leads to

$$
(e+N)^{p^{q}}-((z, 0)+N)^{p^{q}}=0 \in B / N
$$

that is, $(e+N)-\left(\left(z^{p^{q}}, 0\right)+N\right)=0 \in B / N$. Thus, $e \in\left(z^{p^{q}}, 0\right)+$ $N \subseteq A$, the desired contradiction.

Theorem 5. Up to isomorphism, the rings $R$ of characteristic zero that have exactly one proper subring can be classified as the rings satisfying (exactly) one of the following conditions:

(a) $R=\mathbb{Z}[1 / p]$, where $p$ is a prime number (which is uniquely determined by $R$ );

(b) $R=\mathbb{Z} \times \mathbb{F}_{p}$, where $p$ is a prime number (which is uniquely determined by $R$ );

(c) $R=\mathbb{Z}(+) \mathbb{F}_{p}$, where $p$ is a prime number (which is uniquely determined by $R$ ).

Proof. We must identify, up to isomorphism, the rings $R$ such that $\mathbb{Z} \subset R$ is a minimal ring extension which is either (a) integrally closed, (b) decomposed, (c) ramified, or (d) inert. Thanks to [3, Theorem 2.7] (or [13, Theorem 2.4]), we are led to the list of rings in the statement of this result, including the fact that there is no ring $R$ such that $\mathbb{Z} \subset R$ is inert. To obtain the above formulation in (a), recall that the integrally closed minimal ring extensions of $\mathbb{Z}$ are, up to isomorphism, the minimal overrings of $\mathbb{Z}$ (inside $\mathbb{Q}$ ), namely, the rings $\mathbb{Z}[1 / p]$, where $p$ runs over the set of prime numbers.

The above-cited references show that conditions (a), (b), and (c) are mutually exclusive. It remains to establish the uniqueness assertions in those conditions. For (a), note that $p$ is determined as the prime number that has a multiplicative inverse in $R$. The uniqueness assertions in (b) and (c) were established in [3, Theorem 2.7].

We next confront the much more arduous task of classifying the rings of characteristic zero that have exactly two proper subrings. Much of the work below will use the following well known fact. If $A:=R_{1} \times R_{2}$ is a nontrivial direct product of (nonzero) rings $R_{1}$ and $R_{2}$, then, up to isomorphism, the minimal ring extensions of $A$ take one of the forms $E_{1} \times R_{2}$ and $R_{1} \times E_{2}$ where $E_{i}$ is a minimal ring extension of $R_{i}$ for $i=1,2$.

Theorem 6. (1) Up to isomorphism, the rings $R$ of characteristic zero that have exactly two proper subrings can be characterized as the rings satisfying (exactly) one of the following two conditions:

(a) $R:=\mathbb{Z} \times \mathbb{F}_{p^{q}}$, where $p$ and $q$ are (possibly equal) prime numbers (which are uniquely determined by $R$ ); 
(b) for some prime number $p$, there is a ramified extension $B:=\mathbb{Z}(+) \mathbb{F}_{p} \subset R$ with crucial maximal ideal $p \mathbb{Z}(+) \mathbb{F}_{p}$ and, furthermore, $\mathbb{Z}[u]=R$ for all $u \in R \backslash B$.

(2) For each prime number $p$, there exist (nonisomorphic) rings $R_{1}$ and $R_{2}$ such that $B:=\mathbb{Z}(+) \mathbb{F}_{p} \subset R_{i}$ is a ramified extension with crucial maximal ideal $p \mathbb{Z}(+) \mathbb{F}_{p}$ for each $i \in$ $\{1,2\}, R_{1}$ has exactly two proper subrings, and $R_{2}$ has more than two proper subrings.

Proof. (1) Thanks to Theorem 2, we need only address a ring $R$ that can result from a construction described in one of six of the 13 conditions in the statement of Theorem 1 , namely, conditions (i), (ii), (v), (vi), (xi), and (xiii). We will deal with these six conditions in the stipulated order.

Suppose, first, that $R$ is a ring that results from condition (i) in the statement of Theorem 1 . Then, as recalled prior to Proposition 3, there is a prime number $p$ such that $R$ is a minimal overring of the principal ideal domain $B:=\mathbb{Z}[1 / p]$. By [10, Proposition 4.11], $R$ is then (up to isomorphism) $B[1 / b]$ where $b$ is an irreducible element of $B$. Such elements $b$ take the form $b= \pm p^{j} q$, where $j \in \mathbb{Z}$ and $q$ is a prime number that is distinct from $p$. Then

$$
\begin{aligned}
R & =B\left[\frac{1}{b}\right]=\mathbb{Z}\left[\frac{1}{p}\right]\left[\frac{1}{\left(p^{j} q\right)}\right]=\mathbb{Z}\left[\frac{1}{p}\right]\left[\frac{1}{q}\right] \\
& =\mathbb{Z}\left[\frac{1}{(p q)}\right] .
\end{aligned}
$$

However, $\mathbb{Z}[1 / p]$ and $\mathbb{Z}[1 / q]$ are distinct proper subrings of this $R$. In particular, this $R$ satisfies $|[\mathbb{Z}, R]| \geq 4$. (In fact, $|[\mathbb{Z}, R]|=4$.) Thus, condition (i) does not produce any contributions to the list that we are building/verifying.

Next, suppose that $R$ results from condition (ii). Then, as noted in Theorem 5, there is a prime number $p$ such that $R$ is an integrally closed minimal ring extension of either $B_{1}:=$ $\mathbb{Z} \times \mathbb{F}_{p}$ or $B_{2}:=\mathbb{Z}(+) \mathbb{F}_{p}$. We will first show that the first of these alternatives does not contribute to the list that we are building/verifying. As there is no integrally closed minimal ring extension of the form $\mathbb{F}_{p} \subset E_{2}$, it follows from the above comments that, up to isomorphism, $R=E_{1} \times \mathbb{F}_{p}$, where $E_{1}$ is an integrally closed minimal ring extension of $\mathbb{Z}$. As recalled above, this means that there is a prime number $q$ (possibly equal to $p$ ) such that we can take $E_{1}=\mathbb{Z}[1 / q]$. In short, $R=$ $\mathbb{Z}[1 / q] \times \mathbb{F}_{p}$. It will suffice to show that $\mathbb{Z} \subset R$ does not satisfy FIP. In fact, we will show that if $\alpha \in \mathbb{Z}[1 / q] \backslash \mathbb{Z}$ (e.g., $\alpha=1 / q)$ and $\beta \in \mathbb{F}_{p}$ (e.g., $\beta=0$ ), then $u:=(\alpha, \beta) \in R \backslash B_{1}$ is such that $\mathbb{Z} \subset \mathbb{Z}[u]$ does not satisfy FIP. This will follow from [14, Proposition 3.1] after we establish three facts (to show that the cited result is applicable here). The first of these facts is that $\mathbb{Z} \subset \mathbb{Z}[u]$ is not an integral extension; this is clear since condition (ii) ensures that the integral closure of $\mathbb{Z}$ in $R$ is $B_{1}$. The second required fact (actually, slightly more than is needed) is that, for all nonzero elements $v \in R$, there exist integers $a$ and $b$, with $a \neq 0$, such that $a v=b$. (For a proof, pick a positive integer $N$ such that $q^{N} v \in \mathbb{Z} \times \mathbb{F}_{p}$; note that $p q^{N} v=(c, 0) \in \mathbb{Z} \times\{0\}$ for some integer $c ; p q^{N} v-c \in\{0\} \times$ $\mathbb{F}_{p}$; and so $a:=p^{2} q^{N}$ and $b:=p c$ satisfy $a v=b$.) The last required fact is that $\mathbb{Z}$ is a residually finite ring. This completes the proof concerning $B_{1}$.

In the remaining subcase pertinent to condition (ii), $R$ is an integrally closed minimal ring extension of $B:=$ $B_{2}=\mathbb{Z}(+) \mathbb{F}_{p}$. Then by Proposition 3, there is a (uniquely determined) prime number $q \neq p$ such that, up to $B$-algebra isomorphism, $R=\mathbb{Z}[1 / q](+) \mathbb{F}_{p}$. With modest changes to the above argument that applied [14, Proposition 3.1], we can show that $\mathbb{Z} \subset \mathbb{Z}[(1 / q, 0)]$ does not satisfy FIP. Thus, $B_{2}$ also fails to lead to a contribution to the list that we are building. This completes the discussion relative to condition (ii).

Next, note that condition (v) cannot lead to a contribution to our developing list because there is no ring $R$ such that $\mathbb{Z} \subset$ $R$ is inert [3, Theorem 2.7]. We turn to condition (vi). This will lead to the first entry to our list. Indeed, up to isomorphism, the towers $\mathbb{Z} \subset B \subset R$ such that $\mathbb{Z} \subset B$ is decomposed with crucial maximal ideal $M$ and $B \subset R$ is inert with crucial maximal ideal $N$ such that $N \cap \mathbb{Z}=M$ can be characterized via $B:=\mathbb{Z} \times \mathbb{F}_{p}$ and $R:=\mathbb{Z} \times \mathbb{F}_{p^{q}}$, where $p$ and $q$ are (possibly equal) prime numbers. (This can be seen by combining [3, Theorem 2.7], [2, Lemme 1.2], and the classical Galois theory of finite fields. To verify the required behavior of the crucial maximal ideals, observe that $M=p \mathbb{Z}$ and $N=\mathbb{Z} \times 0$.) As for the uniqueness conclusions in the assertion (a), note first that $p$ is the only prime number which, when viewed inside $R$, is a zero-divisor in $R$. It is slightly harder to establish the uniqueness of $q$ without mentioning $M$ and $N$ explicitly, but it can be done as follows (now that the uniqueness of $p$ has been shown). The factor ring $R / M$ is isomorphic to (a unique direct product of the form) $\mathbb{F}_{p} \times F$, where $F$ is a field (actually, $\left.F=\mathbb{F}_{p^{q}}\right)$ whose vector space dimension over $\mathbb{F}_{p}$ is $q$.

By Proposition 4, condition (xi) cannot lead to a contribution to our developing list. It remains only to describe the possible contributions from condition (xiii). Assume, then, that both $\mathbb{Z} \subset B$ and $B \subset R$ are ramified extensions whose crucial maximal ideals, denoted by $M$ and $N$, respectively, satisfy $N \cap \mathbb{Z}=M$. By [3, Theorem 2.7], there exists a uniquely determined prime number $p$ such that we can identify $B=\mathbb{Z}(+) \mathbb{F}_{p}$. Then, necessarily, $M=p \mathbb{Z}$ and $N=$ $p \mathbb{Z}(+) \mathbb{F}_{p}$. The cumbersome conditions from [6, Proposition 3.5(b)] that were alluded to in the statement of condition (xiii) hold automatically in the present context. In other words, $\mathbb{Z} \subset R$ satisfies FIP. The easiest way to see this is to apply the noncumbersome part of [6, Proposition 3.5(b)], the point being that $\mathbb{Z} / M\left(\cong \mathbb{F}_{p}\right)$ is finite. However, our relative lack of understanding of ramified extensions (cf. the third paragraph of [1, Remark 2.11(a)]) has made us settle here for the nonspecificity in assertion (b). One should note that the part of assertion (b) which follows the word "furthermore" is what allows us to conclude that $R$ has exactly two proper subrings. As we showed above that our data satisfy the conditions from [6, Proposition 3.5(b)], it is now clear that the earlier part of assertion (b) simply ensures that we are addressing data satisfying condition (xiii).

(2) We have $B:=\mathbb{Z}(+) \mathbb{F}_{p}$, with $N:=p \mathbb{Z}(+) \mathbb{F}_{p} \in \operatorname{Max}(B)$. Noting that $B / N$ can be identified with $\mathbb{F}_{p}$, put

$$
R_{2}:=B(+) B / N\left(=B(+) \mathbb{F}_{p}=\left(\mathbb{Z}(+) \mathbb{F}_{p}\right)(+) \mathbb{F}_{p}\right) .
$$


By [15], $A \subset R_{2}$ is a minimal ring extension. Its crucial maximal ideal is $N ; N \cap \mathbb{Z}=p \mathbb{Z}=: M$; and this minimal ring extension is ramified (cf. [16, Lemma 2.1]). Consider the ring $D:=(\mathbb{Z}(+) 0)(+) \mathbb{F}_{p}$. It is clear that $D \in\left[\mathbb{Z}, R_{2}\right] \backslash\left\{\mathbb{Z}, B, R_{2}\right\}$, and so $\left|\left[\mathbb{Z}, R_{2}\right]\right|>3$, as required. We turn next to the more difficult construction of a suitable $R_{1}$.

Once again, we are working with $B=\mathbb{Z}(+) \mathbb{F}_{p}$ and $N:=$ $p \mathbb{Z}(+) \mathbb{F}_{p}$. The $B$-algebra $R_{1}$ will be constructed so as to have essentially the same $B$-module structure as $R_{2}$. Specifically, as a set (and then as an additive group with the " $x$ " symbols being viewed as “ $\oplus$ ”), let

$$
R_{1}:=\mathbb{Z} \times \mathbb{F}_{p} x \times \mathbb{F}_{p} z,
$$

where $x$ and $z$ each are generators of (distinct) one-dimensional vector spaces over $\mathbb{F}_{p}$. Next, we define the multiplication in $R_{1}$ by setting $\left(m_{1}+\xi_{1} x+\eta_{1} z\right) \cdot\left(m_{2}+\xi_{2} x+\eta_{2} z\right)$

$$
:=m_{1} m_{2}+\left(m_{1} \xi_{2}+m_{2} \xi_{1}+\eta_{1} \eta_{2}\right) x+\left(m_{1} \eta_{2}+m_{2} \eta_{1}\right) z
$$

for all $m_{1}, m_{2} \in \mathbb{Z}$ and all $\xi_{1}, \xi_{2}, \eta_{1}, \eta_{2} \in \mathbb{F}_{p}$. (Of course, expressions such as $m_{i} \xi_{j}$ and $m_{i} \eta_{j}$ are interpreted by using the additive structure of $\mathbb{F}_{p}$.)

It is straightforward (albeit somewhat tedious) to verify that $R_{1}$ is a ring (having $1 \in \mathbb{Z}$ as its multiplicative identity element). Moreover, the function $B \rightarrow R_{1}$, given by $(m, \xi) \mapsto$ $m+\xi x$ for all $m \in \mathbb{Z}$ and $\xi \in \mathbb{F}_{p}$, is an injective (unital) ring homomorphism, thus allowing us to view $B$ as a subring of $R_{1}$. A more intuitive understanding of the structure of $R_{1}$ as a $B$-algebra is that we required the multiplication to satisfy the axiomatic restrictions and the additional relations $x^{2}=0$, $x z=0$, and $z^{2}=x$. (As $(0,1) \in B$ has been identified with $1 x=x$, we see that the last two relations differ from their analogues in $R_{2}$.)

Recall that $\mathbb{Z} \subset B$ is a ramified extension with crucial maximal ideal $M=p \mathbb{Z}$. We claim that $B \subset R_{1}$ is a ramified extension with crucial maximal ideal $N$ (such that $N \cap \mathbb{Z}=M$ ). Consider the set (described additively as) $N_{1}:=p \mathbb{Z} \oplus \mathbb{F}_{p} x \oplus \mathbb{F}_{p} z \subseteq R_{1}$. It is straightforward to verify that $N_{1}$ is a prime ideal of $R_{1}$ such that $N_{1} \cap B=$ $N$. As $R_{1}$ is integral over $B$, the fact that $N$ is a maximal ideal of $B$ ensures that $N_{1}$ is a maximal ideal of $R_{1}$. It is also straightforward to verify that $\left(N_{1}\right)^{2} \subseteq N \subset N_{1}$ and that the canonical map $\left(\mathbb{F}_{p} \cong \mathbb{Z} / M \cong\right) B / N \rightarrow R_{1} / N_{1}$ is an isomorphism. In addition, one can verify that $N$ is an ideal of $R_{1}$ (since $p \mathbb{F}_{p}=0$ and $x z=0$ ) and $R_{1} / N$ is a two-dimensional vector space over $B / N$. Therefore, according to [12, Theorem $4.2(\mathrm{c})]$, the claim has now been proved.

It remains to show that $R_{1}$ has only two proper subrings. It is enough to show that if $u \in R_{1} \backslash B$, then $\mathbb{Z}[u]=R_{1}$. Without loss of generality, $u=\xi x+\eta z$ for some $\xi, \eta \in \mathbb{F}_{p}$. Note that $\eta \neq 0$ since $u \notin B$. Thus, the above rules for multiplication lead to $u^{2}=\eta^{2} x \neq 0$. In particular, $\mathbb{Z}[u]$ contains the element $\nu\left(\eta^{2} x\right)$ for each integer $\nu$. In other words, $\mathbb{F}_{p}\left(\eta^{2} x\right) \subseteq \mathbb{Z}[u]$. But $\mathbb{F}_{p}\left(\eta^{2} x\right)=\left(\eta^{2} \mathbb{F}_{p}\right) x=\mathbb{F}_{p} x$. Now, since $\mathbb{F}_{p} x \subseteq \mathbb{Z}[u]$, we get that $\eta z=u-\xi x \in \mathbb{Z}[u]$. Since $\eta \neq 0$, we can see, by taking integer multiples as above, that $\mathbb{F}_{p} z=\mathbb{F}_{p}(\eta z) \subseteq \mathbb{Z}[u]$. It is now evident that $R_{1} \subseteq \mathbb{Z}[u]$, which completes the proof.
One can fairly state that the formulation of assertion (b) in part (1) of the preceding theorem means that we have given a characterization but not a classification of the rings $R$ in question. As noted in the proof, this lack of a classification is due solely to our current inability to classify certain ramified extensions (up to isomorphism). This feature of having a characterization, but not a classification, will recur when we treat the corresponding problems for base rings of positive characteristic; there, too, the sole reason for a lack of a classification will be our incomplete information concerning certain ramified extensions.

It was shown in [9, Proposition V.1] that if a nonzero ring $R$ has only finitely many subrings and $\operatorname{char}(R) \neq 0$, then $R$ is a finite ring. Having dispatched the case of characteristic 0 (and the trivial case of the zero ring), we henceforth devote our attention to nonzero finite rings. The first technical lemma leading to our main results on finite rings is given next. It will be applicable because any (nonzero) finite ring is zerodimensional.

Proposition 7. Let $A$ be a (nonzero) zero-dimensional ring. Then there does not exist a ring $B$ such that $A \subset B$ is an integrally closed minimal ring extension.

Proof. Suppose the assertion fails. Let $M$ denote the crucial maximal ideal of an integrally closed minimal ring extension $A \subset B$. As $A \subset B$ is a minimal ring extension which is not integral, it follows from [2, Théorème 2.2(ii)] that there does not exist a prime ideal $Q$ of $B$ such that $Q \cap A=M$. However, $M$ is a minimal prime ideal of $A(\operatorname{since} \operatorname{dim}(A)=0)$, and so by an application of Zorn's Lemma [5, Exercise 1, page 41], such a prime ideal $Q$ does exist, the desired contradiction.

Thanks to Proposition 7 and the comments that preceded it, we may now focus on integral minimal ring extensions of finite rings. The next result is in the same spirit as Proposition 3. To further motivate it, first recall from [2, Lemme 1.2] that if $p$ is a prime number, the inert extensions of $\mathbb{Z} / p \mathbb{Z}$ (i.e., of $\mathbb{F}_{p}$ ) are the minimal field extensions of $\mathbb{F}_{p}$. The next result shows that if $\alpha>1$ (and $p$ is a prime number), then the base ring $\mathbb{Z} / p^{\alpha} \mathbb{Z}$ behaves differently. It will also be useful to note that the hypothesis of the next result accommodates base rings such as $\mathbb{F}_{p}[X] /\left(X^{2}\right)$. Indeed, it accommodates base rings that are arbitrary special principal ideal rings (other than fields).

Proposition 8. Let $(A, M)$ be a quasi-local zero-dimensional principal ideal ring which is not a field. Then there does not exist a ring $B$ such that $A \subset B$ is an inert extension.

Proof. Observe that $M$ is the only prime ideal of $A$. As $A$ is a principal ideal ring, there exists $x \in A$ such that $M=A x$. As $A$ is not a field, $M \neq 0$, and so $x \neq 0$. Suppose the assertion fails. Then $M$ is the crucial maximal ideal of some inert extension $A \subset B$. The "inert" condition ensures that $(B, M)$ is quasi-local. Next, choose any element $e \in B \backslash A$. Then $B=A[e]$, by the minimality of $A \subset B$. As $x e \in M B=M$, there exists $a \in A$ such that $x e=a x$, and so $x(a-e)=0$. Since $x \neq 0, a-e$ cannot be a unit of $B$; that is, $a-e \in M$. 
Hence, $e=a-(a-e) \in A+M=A$, the desired contradiction.

Remark 9. Although Proposition 8 has been stated in a form that will be useful below, it may be of interest to record the following generalization of it. If $A$ is a (nonzero) zerodimensional ring such that $A_{M}$ is a principal ideal ring and $M A_{M} \neq 0$ for each $M \in \operatorname{Max} A$, then there does not exist a ring $B$ such that $A \subset B$ is an inert extension. For a proof by contradiction, take $M$ to be the crucial maximal ideal of an inert extension $A \subset B$. Next, apply [12, Proposition 4.6] to replace $A \subset B$ with $\left(A_{M}, M A_{M}\right) \subset B_{M}$. Then repeat the proof of Proposition 8 to get the desired contradiction.

In contrast to Propositions 7 and 8, Proposition 10 shows that there does exist a ring $B$ with the relevant property (and that it is unique up to isomorphism). As an application, it also shows a way in which the base ring $\mathbb{Z} / p^{\alpha} \mathbb{Z}$ behaves regardless of whether the positive integer $\alpha$ exceeds 1 . In view of Proposition 8, this highlights an important difference in the behavior of the "inert" and "decomposed" concepts.

Proposition 10. Let $(A, M)$ be a quasi-local zero-dimensional principal ideal ring. Then, up to A-algebra isomorphism, the unique ring $B$ such that $A \subset B$ is a decomposed extension is $B=A \times A / M$.

Proof. By [3, Lemma 2.2] (or the first paragraph of the proof of [13, Corollary 2.5]), $A \subset A \times A / M$ is a minimal ring extension. As this minimal ring extension is integral, it follows from [2, Théorème 2.2(ii)] that its crucial maximal ideal is its conductor, namely, $M$ (viewed as $\mathscr{M}:=M \times 0 \subset$ $A \times A / M)$. Since $(A \times A / M) / \mathscr{M} \cong A / M \times A / M$, we see that $A \subset A \times A / M$ is a decomposed extension.

Conversely, suppose that $A \subset B$ is a decomposed extension. We will prove that $B$ is $A$-algebra isomorphic to $A \times A / M$. By [2, Lemme 1.2], we may assume that $A$ is not a field. In particular, $M \neq 0$. Since $R$ is a principal ideal ring, there exists $x \in A$ such that $M=A x$. As $M \neq 0$, we get $x \neq 0$. As $M$ is the only prime ideal of $A$, it consists of all the nilpotent elements of $A$. Let $k(\geq 2)$ be the index of nilpotence of $x$. Clearly, $M$ is a nilpotent ideal whose index of nilpotence is $k$. Next, notice that $A$ is an Artinian ring, since it is a zerodimensional Noetherian ring. Since $B$ is finitely generated as an $A$-module, it follows that $B$ is also an Artinian ring. By a fundamental structure theorem [17, Theorem 3, page 205], $B$ is (essentially uniquely expressible as) the direct product of finitely many (nonzero Artinian quasi-) local rings, say, $B=\prod_{i=1}^{n} C_{i}$. In fact, $n=2$ since the "decomposed" hypothesis ensures that $B$ has exactly two maximal ideals, say $N_{1}$ and $N_{2}$. The "decomposed" hypothesis also ensures that $N_{1} \cap N_{2}=M$; and the canonical ring (field) homomorphism $A / M \rightarrow B / N_{i}$ is an isomorphism for $i=1,2$. With $\mathcal{N}_{i}$ denoting the unique maximal ideal of $C_{i}$, there is no harm in taking $N_{1}=\mathcal{N}_{1} \times C_{2}$ and $N_{2}=C_{1} \times \mathcal{N}_{2}$. It will be useful later to notice that $C_{i} / \mathcal{N}_{i} \cong B / N_{i}(\cong A / M)$ for $i=1,2$.

Next, note that $A$ is a special principal ideal ring (in short, an SPIR), in the sense of [17, page 245]. (The literature contains a variety of definitions of an SPIR, but those definitions are all equivalent.) Consequently, each nonzero proper ideal of $R$ is of the form $M^{j}$ for some integer $j$ such that $1 \leq j \leq k-1$ [17, page 245]. Then, for each $i=1,2$, the First Isomorphism Theorem shows that the composition of the inclusion map $R \hookrightarrow S$ with the canonical projection map $S \rightarrow C_{i}$ has an image which is a subring of $C_{i}$ that is isomorphic as an $A$-algebra to $A / M^{\lambda_{i}}$ for some nonnegative integer $\lambda_{i} \leq k$. There is no harm in viewing $A / M^{\lambda_{i}}$ as a subring of $C_{i}$ for $i=1,2$. Observe that the canonical map $\varphi$ : $A \rightarrow A / M^{\lambda_{1}} \times A / M^{\lambda_{2}}$ is an injection (since the composition of $\varphi$ with the inclusion map $A / M^{\lambda_{1}} \times A / M^{\lambda_{2}} \hookrightarrow C_{1} \times C_{2}$ is the inclusion map $A \hookrightarrow B)$. For each $a \in A$, we have $\varphi(a)=\left(a+M^{\lambda_{1}}, a+M^{\lambda_{2}}\right)$. Put $\lambda:=\max \left(\lambda_{1}, \lambda_{2}\right)$. Then $\varphi\left(x^{\lambda}\right)=(0,0)$. Since $\varphi$ is an injection, $\lambda \geq k$. Thus, either $\lambda_{1}$ or $\lambda_{2}$ is $k$. Without loss of generality $\lambda_{1}=k$. Hence, we can view $A\left(\cong A / 0=A / M^{k}=A / M^{\lambda_{1}}\right)$ as a subring of $C_{1}$.

We claim that $A$ is not a proper subring of $C_{1}$. To see this, suppose, on the contrary, that $A \subset C_{1}$. Using $\varphi$ to identify $A$ with its image in $B$, we then get $A \subseteq A \times C_{2} \subset C_{1} \times C_{2}=B$, and so the minimality of $A \subset B$ yields that $A=A \times C_{2}$. However, this gives a contradiction, since $A$ has only one prime ideal while $A \times C_{2}$ has two distinct prime ideals. This contradiction proves the claim. Thus, $B=A \times C_{2}$. It remains to show that $C_{2} \cong A / M$ as algebras over $A / M$.

We have $N_{1}=M \times C_{2}$ and $N_{2}=A \times \mathcal{N}_{2}$. It follows that

$$
\begin{aligned}
M & =N_{1} \cap N_{2}=(M \cap A) \times\left(C_{2} \cap \mathcal{N}_{2}\right)=M \times \mathcal{N}_{2} \\
& \subseteq A \times C_{2}=B .
\end{aligned}
$$

Of course, when viewed inside $A \times C_{2}$, each element $y$ of $M$ is identified with $\left(y, z_{y}\right)$, where $z_{y} \in C_{2}$ is the image of $y$ under the canonical projection map $S \rightarrow C_{2}$. Thus, viewing matters in $A \times C_{2}$, we have

$$
\left\{\left(y, z_{y}\right) \in S \mid y \in M\right\}=M=M \times \mathscr{N}_{2} .
$$

Each $y \in M$ occurs as the first coordinate of only one element in the last-displayed set, and so $\mathscr{N}_{2}$ must be a singleton set. Hence, $\mathcal{N}_{2}=0$; that is, $C_{2}$ is a field. Therefore, $C_{2} \cong C_{2} / 0=$ $C_{2} / \mathcal{N}_{2} \cong A / M$, as desired.

The next result classifies certain ramified extensions. Its proof served us as motivation for the second construction that was used in the proof of Theorem 6(2).

Proposition 11. Let $B:=\mathbb{F}_{p}[X] /\left(X^{2}\right)$, where $p$ is a prime number. As usual, write $B=\mathbb{F}_{p} \oplus \mathbb{F}_{p} x$, where $x^{2}=0 \neq$ $x:=X+\left(X^{2}\right)$. Then, up to $B$-algebra isomorphism, there are exactly two rings $R_{i}(i=1,2)$ such that $B \subset R_{i}$ is a ramified extension. One of these rings, say $R_{1}$, can be constructed as the $B$-algebra whose additive structure as a vector space over $\mathbb{F}_{p}$ is given by $R_{1}=B \oplus \mathbb{F}_{p} z$ for some nonzero element $z$ and whose multiplication is determined by the relations $x z=0$ and $z^{2}=x\left(\right.$ and $\left.x^{2}=0\right)$. The other ring $R_{2}$ can be taken as $B(+) \mathbb{F}_{p}$. Moreover, $R_{1}$ has exactly two proper subrings, while $R_{2}$ has more than two proper subrings (and so $R_{1}$ is not isomorphic to $R_{2}$ ). 
Proof. To shape the constructions of the rings $R_{i}$, consider any ramified extension $R$ of $B$. The crucial maximal ideal of $B \subset R$ is necessarily then the unique prime ideal of $B$; namely, $N:=\mathbb{F}_{p} x$. As recalled in the proof of Proposition 10, the fact that $B \subset R$ is an integral minimal ring extension gives that $N=(B: R)$. In particular, $N$ is an ideal of $R$. Moreover, since $B \subset R$ is ramified and $R$ is quasi-local, it follows that $R$ must be quasi-local, say with maximal ideal $Q$, such that $Q^{2} \subseteq N \subset Q, R / Q \cong B / N\left(\cong \mathbb{F}_{p}\right)$, and $R / N \cong(B / N)[X] /\left(X^{2}\right) \cong \mathbb{F}_{p}[X] /\left(X^{2}\right)$. Hence $\operatorname{dim}_{\mathbb{F}_{p}}(R)=$ $\operatorname{dim}_{\mathbb{F}_{p}}(R / N)+\operatorname{dim}_{\mathbb{F}_{p}}(N)=2+1=3$, and so $\operatorname{dim}_{\mathbb{F}_{p}}(Q)=$ $\operatorname{dim}_{\mathbb{F}_{p}}(R)-\operatorname{dim}_{\mathbb{F}_{p}}(R / Q)=3-1=2$. Therefore, taking $z$ to be any element of $Q \backslash N$, we have $Q=N \oplus \mathbb{F}_{p} z=\mathbb{F}_{p} x \oplus \mathbb{F}_{p} z$. Observe that $z^{2} \in Q^{2} \subseteq N$.

We claim that $x z=0$. In fact, $x z \in N B=N$ and so $x z=a x$ for some $a \in \mathbb{F}_{p}$. Thus $x(a-z)=0$. Since $x \neq 0$, it follows that $a-z$ is not invertible in $R$. As $z^{4}=\left(z^{2}\right)^{2} \epsilon$ $\left(Q^{2}\right)^{2} \subseteq N^{2}=0$, we see that $z$ is nilpotent. Hence, $a$ is not invertible (in $R$ ). Since $a$ is a member of the field $\mathbb{F}_{p}$, we get $a=0$, whence $x z=0$, thus proving the above claim. Note also that $z^{3}=0$ since $z^{3}=z^{2} z \in Q^{2} z \subseteq N z=\mathbb{F}_{p} x z=0$.

Since $\mathbb{F}_{p} \cap Q=0$ and $\operatorname{dim}_{\mathbb{F}_{p}}(R)=\operatorname{dim}_{\mathbb{F}_{p}}\left(\mathbb{F}_{p}\right)+\operatorname{dim}_{\mathbb{F}_{p}}(Q)(<$ $\infty)$, it follows that the linear structure of $R$ must be given by $R=\mathbb{F}_{p} \oplus Q=\mathbb{F}_{p} \oplus \mathbb{F}_{p} x \oplus \mathbb{F}_{p} z$. Given this explicit linear structure and the specific facts that we have found about multiplication in $R$, there are now two general ways to describe a set of necessary conditions for the $B$-algebra $R$. One would impose the following set of relations: $x^{2}=0, x z=0$, and $z^{2}=0$. This set of conditions determines the $B$-algebra $R_{2}:=B(+) \mathbb{F}_{p}$. It is known that $B \subset R_{2}$ is ramified (see [15] and [16, Lemma 2.1]), and so this given set of relations should not be augmented in a possible search for other possible $R$. To show that $R_{2}$ has more than two proper subrings, one need only tweak an argument that was given in the proof of Theorem 6(2): consider $D:=\mathbb{F}_{p} \oplus 0 \oplus \mathbb{F}_{p} z$.

However, there is one other general way to prescribe the multiplication of $R=\mathbb{F}_{p} \oplus \mathbb{F}_{p} x \oplus \mathbb{F}_{p} z$, namely, as follows: $x^{2}=0, x z=0$, and $z^{2}=b x$, where $b$ is some nonzero element of $\mathbb{F}_{p}$. We next explain that any such prescription does give a $B$-algebra and that any two such prescriptions (using different values of the nonzero element $b$ ) give isomorphic $B$-algebras. The first of these tasks can be handled classically, as we have merely stipulated how to multiply the basis elements of a vector space. (Lacking that vectorial context led us to the more complicated argumentation given for the somewhat similar construction in the proof of Theorem 6(2).) Let $R_{1}$ denote the $B$-algebra that is constructed when we use $b:=1$. The second task is now handled by noticing that any other nonzero element $b$, say $\beta \in \mathbb{F}_{p} \backslash\{0\}$, gives a $B$-algebra that is isomorphic to $R_{1}$. What it does give is a multiplication that is determined on the elements of the $\mathbb{F}_{p}$-basis $\{1, x, z\}$ of the $\mathbb{F}_{p}$-algebra by $x^{2}=0, x z=0$, and $z^{2}=\beta x$. Consider the induced multiplication on the basis consisting of $1, x_{1}:=\beta^{3} x$, and $z_{1}:=\beta z$. One easily finds that $x_{1}^{2}=0, x_{1} z_{1}=0$, and $z_{1}^{2}=\beta^{2} z^{2}=\beta^{2} \beta x=\beta^{3} x=x_{1}$. Thus, the construction using $\beta$ has produced an algebra that is isomorphic (via the change of bases from $\{1, x, z\}$ to $\left.\left\{1, x_{1}, z_{1}\right\}\right)$ to the algebra that was constructed using $b:=1$.

Only two tasks remain: to prove that $B \subset R_{1}$ is ramified and that $R_{1}$ has only two proper subrings. To accomplish the first of these, we will invoke [12, Theorem 4.2]. For that, it suffices (since $\operatorname{Max}(B)=\{N\}$ ) to show the following: $Q:=N+$ $\mathbb{F}_{p} z\left(=\mathbb{F}_{p} x \oplus \mathbb{F}_{p} z\right)$ is a maximal ideal of $R$ such that $Q^{2} \subseteq N \subset$ $Q, \operatorname{dim}_{B / N}(R / N)=2$, and the canonical map $B / N \rightarrow R / Q$ is an isomorphism. All of the preceding assertions are easily verified and so we omit those details. Finally, to show that $R_{1}$ has only two proper subrings, it suffices to show that if $u \in R \backslash$ $B$, then $\mathbb{F}_{p}[u]=R$. Without loss of generality, $u=\xi x+\eta z$ for some $\xi, \eta \in \mathbb{F}_{p}$. To complete this proof, it suffices to repeat the argument in the final paragraph of the proof of Theorem 6, with that proof's coefficient ring $\mathbb{Z}$ now being modified to $\mathbb{F}_{p}$ for the purposes of this proof.

Note that the idealization used in Proposition 11 can also be described as $R_{2}=\mathbb{F}_{p}[X, Z] /\left(X^{2}, X Z, Z^{2}\right)$. In the same spirit, one could describe the ring $R_{1}$ from that result as $\mathbb{F}_{p}[X, Z] /\left(X^{2}, X Z, Z^{2}-X\right)$. This description of $R_{1}$ is admittedly more compact than the earlier one, but that earlier description seemed more suitable in presenting the proof of Proposition 11.

Part (a) of the next result was developed in correspondence with Jay Shapiro and is included here with his kind permission.

Proposition 12. Let $A:=\mathbb{Z} / p^{\alpha} \mathbb{Z}$, where $p$ is a prime number and $\alpha \geq 2$ is an integer. Let $M:=p A$, the maximal ideal of $A$. Then, consider the following:

(a) Let $B$ be a ring such that $A \subset B$ is a ramified extension. Let $Q$ denote the maximal ideal of $B$. Then there exists $y \in Q \backslash M$ such that $B=A[y], y^{2} \in M$, and $p y \in M$. In fact, $p^{\alpha-1} y=0$. Moreover, there exist units $u$ and $v$ of $A$ and uniquely determined integers $i$ and $j$ such that $y^{2}=u p^{i}, p y=v p^{j}$, and $1 \leq i, j \leq \alpha$. Also, $\left(u-v^{2}\right) p^{2 j}=0$. If $i<\alpha-2$, then $i$ is even and $j=$ $(i+2) / 2$ (so $2 \leq j<\alpha / 2)$. If $i \geq \alpha-2$, then $j \geq \alpha / 2$.

(b) Let $B$ be a ring such that $A \subset B$ is a ramified extension. Let $y \in B$ be as in (a). Let $H:=\{r y \in B \mid 0 \leq$ $r \leq p-1$ in $\mathbb{Z}$. Then $H$ is a subset (not necessarily an additive subgroup) of $B$ of cardinality $p$. Moreover, the assignment $(a, k y) \mapsto a+k y$ gives a bijection $A \times H \rightarrow B$ (which does not necessarily preserve any algebraic structure).

(c) If $B$ is a ring such that $A \subset B$ is a ramified extension, then $|B|=p^{\alpha+1}$.

(d) $A \subset A(+) \mathbb{F}_{p}$ is a ramified extension.

(e) Up to isomorphism, $\mathbb{Z} / 4 \mathbb{Z}$ has exactly two ramified extensions, namely, the ring $B_{1}:=\mathbb{Z} / 4 \mathbb{Z}(+) \mathbb{F}_{2}$ given by $(d)$ and the ring $B_{2}:=(\mathbb{Z} / 4 \mathbb{Z})[X] /\left(X^{2}-2,2 X\right)$.

(f) Suppose that $p$ is an odd prime number. Then, up to isomorphism, $A:=\mathbb{Z} / p^{2} \mathbb{Z}$ has exactly three ramified extensions, namely, the ring $B_{a}:=A(+) \mathbb{F}_{p}$ given by $(d)$, the ring $B_{b}:=A[X] /\left(p X, X^{2}-p\right)$, and the ring 
$B_{c}:=A[X] /\left(p X, X^{2}-d p\right)$, where $d$ is a (ny) quadratic nonresidue of $p$.

Proof. (a) By [13, Proposition 2.12], there exists $y \in Q \backslash M$ such that $B=A[y], y^{2} \in M, y^{3} \in M$, and $(A: B)=M$. In particular, $p y \in M B=M$. As $A$ is an SPIR whose unique prime ideal is generated by $p$, it follows from [17, page 245] that there exist uniquely determined integers $i$ and $j$ and units $u$ and $v$ of $A$ such that $y^{2}=u p^{i}, p y=v p^{j}$, and $1 \leq i, j \leq \alpha$. Thus

$$
v^{2} p^{2 j}=\left(v p^{j}\right)^{2}=(p y)^{2}=p^{2} y^{2}=p^{2}\left(u p^{i}\right)=u p^{i+2} .
$$

If $i<\alpha-2$, then the right-hand side of the displayed equation is nonzero (again, by [17, page 245]), and thus so is the lefthand side, whence $2 j=i+2$ (again, by [17, page 245]), so that $i=2 j-2$ and $j=(i+2) / 2$. In particular, if $i<\alpha-2$, then $i$ is even, $2 \leq j<\alpha / 2$, and $\left(u-v^{2}\right) p^{2 j}=u p^{2 j}-v^{2} p^{2 j}=$ $u p^{i+2}-v^{2} p^{2 j}=0$. On the other hand, if $i \geq \alpha-2$, then the right-hand side of the displayed equation is 0 , hence so is its left-hand side, whence $2 j \geq \alpha$ and $j \geq \alpha / 2$.

It remains only to prove that $p^{\alpha-1} y=0$. First, note that $y$ is nilpotent since $y^{2}$ is a member of the only prime ideal of $A$. (In general, $y^{2 \alpha}=\left(y^{2}\right)^{\alpha} \in M^{\alpha}=0$.) Recall that $p y=v p^{j}$. As $p^{\alpha-1} y=p^{\alpha-2}(p y)$ and $p^{\alpha}=0$, it suffices to show that $j \geq 2$. Suppose, on the contrary, that $p y=v p$. Then $p(v-y)=0$. As the nilpotence of $y$ implies that $v-y$ is a unit of $B$, it follows that $p=0$, contradicting $\alpha>1$.

(b) Let $k \in \mathbb{Z}$ with $1 \leq k<p^{\alpha}$. By the division algorithm, there exist nonnegative integers $q$ and $r$ such that $k=q p+r$ and $r<p^{\alpha}$. Thus, $k y=q(p y)+r y$ can be expressed as the sum of an element of $A$ with an element of $H$. We will show that this expression is unique.

We claim that there cannot exist nonnegative integers $\lambda<$ $\mu<p$ such that $(\mu-\lambda) y \in A$. For a proof, suppose, on the contrary, that $\nu:=\mu-\lambda$ satisfies $\nu y \in A$. Since $1 \leq \nu \leq p-1$, $\nu$ and $p$ are relatively prime, and so there exist integers $n_{1}, n_{2}$ such that $n_{1} v+n_{2} p=1$. Hence, $n_{1}(v y)+n_{2}(p y)=y$. As $v y$ and $p y$ are elements of $A$, it follows that $y \in A$, the desired contradiction. This proves the above claim.

Now suppose that $a_{1}+k_{1} y=a_{2}+k_{2} y$, where $a_{1}, a_{2} \in A$ and $0 \leq k_{1} \leq k_{2}<p$. As $\left(k_{2}-k_{1}\right) y=a_{1}-a_{2} \in A$, it follows from the above claim that $k_{1}=k_{2}$ (hence $k_{1} y=k_{2} y$ ) and $a_{1}=a_{2}$. This proves the above uniqueness assertion. It also shows that $|H|=p$ and establishes the asserted bijection.

(c) One proof of (c) is via (b), for $|B|=|A \times H|=$ $|A| \cdot|H|=p^{\alpha} p=p^{\alpha+1}$. We next give an alternate proof. Let $Q$ denote the maximal ideal of $B$. Since $A \subset B$ is ramified, the canonical map $A / M \rightarrow B / Q$ is an isomorphism and $B / M \cong(A / M)[X] /\left(X^{2}\right)\left(\cong \mathbb{F}_{p}[X] /\left(X^{2}\right)\right)$. Therefore, by Lagrange's Theorem, $|B|=|B / M| \cdot|M|=p^{2} p^{\alpha-1}=p^{\alpha+1}$.

(d) By $[15], B:=A(+) \mathbb{F}_{p}$ is a minimal ring extension of $A$; and this extension is ramified (i.e., subintegral) by [16, Lemma 2.1].

(e), (f) Let $B$ be a ramified extension of $\mathbb{Z} / p^{2} \mathbb{Z}$ and let $Q$ denote the maximal ideal of $B$. We adopt the other data from (a) (now with $A=\mathbb{Z} / p^{2} \mathbb{Z}$ ). Recall from (a) that $p^{\alpha-1} y=0$. As $\alpha=2$ here, $p y=0$. Next, since $y^{3} \in M$, it follows as above via [17, page 245] that $y^{3}=w p^{k}$ for some unit $w$ of $A$ and some (uniquely determined) positive integer $k \leq 2$. It cannot be the case that $i=k=1$. (Otherwise, $y^{2}=u p$ and $y^{3}=w p$, with $u$ and $w$ suitable units of $A$ as above, and so $p=$ $w^{-1}(w p)=w^{-1} y^{3}=w^{-1} y^{2} y=w^{-1}(u p) y=\left(w^{-1} u\right) \cdot 0=0, \mathrm{a}$ contradiction since $\alpha>1$.) On the other hand, if $i=2$, then $k=2$ (the point being that if $y^{2}=0$, then $\left.y^{3}=0\right)$. Thus, $(i, k)$ is either $(2,2)$ or $(1,2)$. In any event, $k=2$, and so $y^{3}=0$. In summary, we have $p y=0, y^{3}=0$, and $y^{2}$ is either 0 or of the form $u p$ (for some unit $u$ of $A$ ).

We first consider (e), where $p=2$. Then, since the set of units of $A$ is $\{1,-1\}$, we have that $y^{2}$ is either 0 or $u p= \pm p=$ $\pm 2=2$. By (b) and (c), $B=\{0,1,2,3, y, y+1, y+2, y+3\}$ has cardinality 8 . Since the algebraic structure of $B$ is determined by the values of $y^{2}$ and $2 y(=0$ ), it follows that (up to isomorphism) there are at most two ramified extensions $B$ of $A$. The first of these candidates is $B_{1}:=(\mathbb{Z} / 4 \mathbb{Z})[X] /\left(X^{2}, 2 X\right)$, which arises from the identities $y^{2}=0$ and $2 y=0$. Note that $B_{1}$ is isomorphic to $\mathbb{Z} / 4 \mathbb{Z}(+) \mathbb{F}_{2}$, which we know, by $(\mathrm{d})$, is a ramified extension of $\mathbb{Z} / 4 \mathbb{Z}$ (where $y \in 0(+) \mathbb{F}_{2}$ can be taken to be $(0,1))$. Hence, $B_{1}$ is a ramified extension of $\mathbb{Z} / 4 \mathbb{Z}$.

The remaining candidate is $B_{2}:=(\mathbb{Z} / 4 \mathbb{Z})[X] /\left(X^{2}-\right.$ $2,2 X)$, which arises from the identities $y^{2}=2$ and $2 y=0$. While it was rather obvious that $A \subset B_{1}$, we next include a proof that $A \subset B_{2}$. To view $A \subseteq B_{2}$, we will show that the canonical $A$-algebra homomorphism $A \rightarrow B_{2}$ is an injection; in other words, we claim that $A \cap\left(X^{2}-2,2 X\right)=0$. Suppose, on the contrary, that $a=\left(X^{2}-2\right) f+2 X g$ for some $a \in A \backslash\{0\}$ and some $f, g \in A[X]$. Equating constant terms shows that $a \in$ $M(=2 A)$. As $a \neq 0$, it follows that the constant term of $f$ must be a unit of $A$. Hence, the coefficient of $X^{2}$ in $\left(X^{2}-2\right) f+2 X g$ is a unit of $A$. This is a contradiction (since $a=a+0 X^{2}$ ). This proves the above claim. We leave to the reader a similar argument (focusing on the coefficient of $X$ ) which reveals that $X \notin A+\left(X^{2}-2,2 X\right)$. Thus, $y:=X+\left(X^{2}-2,2 X\right)$ satisfies $A \subset B_{2}=A[y]=A+A y$, with $y^{2}=2$ and $2 y=0$. Next, note that the set of nilpotent elements of $B_{2}$, namely, its unique prime ideal, is $Q=\{0,2, y, y+2\}$. (The same could be said of $B_{1}$.) By verifying the conditions in [12, Theorem $4.2(\mathrm{c})]$, it is straightforward to verify that $B_{2}$ is a ramified extension of $\mathbb{Z} / 4 \mathbb{Z}$. (In detail, $\left|B_{2} / Q\right|=\left|B_{2}\right| /|Q|=8 / 4=2$, so $B_{2} / Q \cong \mathbb{F}_{2} \cong A / M ; \operatorname{dim}_{A / M}(B / M)=2$ since $|B / M|=$ $|B| /|M|=8 / 2=4=|A / M|^{2}$; and $Q^{2}=\{0,2\} \subseteq M \subset Q$.)

It remains only to verify that $B_{1}$ and $B_{2}$ are not isomorphic. For each of these rings $B_{i}$, the only elements of $B_{i}$ that can play the role of $y$ in (a) are $y$ and $y+2$; and $Q \backslash M=$ $\{y, y+2\}$. In $B_{1}$ (resp., $B_{2}$ ), each element of $Q \backslash M$ has index of nilpotence 2 (resp., 3). This completes the proof of (e).

We turn to (f), where $p$ is an odd prime and $A:=$ $\mathbb{Z} / p^{2} \mathbb{Z}$. The first candidate for a ramified extension of $A$ is $B_{a}:=A[X] /\left(X^{2}, p X\right)$, which arises from the identities $y^{2}=0$ and $p y=0$. As above, we see via (d) that $B_{a}$ is a ramified extension of $A$ since $B_{a} \cong A(+) \mathbb{F}_{p}$. Any other (nonisomorphic) candidate for a ramified extension of $A$ must arise from the identities $y^{2}=u p$ and $p y=0$ for some unit $u$ of $A$. This candidate can be viewed as $C_{u}:=$ $A[X] /\left(X^{2}-u p, p X\right)$. Ostensibly, $u$ could be the coset of $p^{2} \mathbb{Z}$ in 
$\mathbb{Z}$ that is represented by any positive integer $m \leq p^{2}-1$ which is not divisible by $p$. However, it suffices to further restrict $m \leq p-1$, for if the division algorithm gives $m=q p+r$, then $p^{2}=0 \in A$ leads to $C_{m}=C_{r}$ since $X^{2}-m p=\left(X^{2}-r p\right)+q p^{2}=$ $X^{2}-r p \in A[X]$. As the above illustrates, it will be convenient (and harmless) to blur the distinction between $u$ and its coset representative $m$, so we will view $u \in \mathcal{S}:=\{1, \ldots, p-1\}$. As is well known (cf. [18, Theorem 9.1]), exactly half of the elements in the set $\delta$ are quadratic nonresidues of $p$ (and the other half are quadratic residues of $p$ ). To finish the proof of (f), it suffices to establish the following five facts (where $u, u_{1}, u_{2} \in \mathcal{S}$ ): each $C_{u}$ is a ramified extension of $A ; C_{u}$ is not isomorphic to $B_{a}$; if $u_{1}$ and $u_{2}$ each are quadratic residues of $p$, then $C_{u_{1}} \cong C_{u_{2}}$; if $u_{1}$ and $u_{2}$ each are quadratic nonresidues of $p$, then $C_{u_{1}} \cong C_{u_{2}}$; and if $u_{1}$ is a quadratic residue of $p$ and $u_{2}$ is a quadratic nonresidue of $p$, then $C_{u_{1}}$ and $C_{u_{2}}$ are not isomorphic.

Let $u \in \mathcal{S}$. By reworking the above analysis of $B_{2}$ in the proof of (e), we can view $A \subset C_{u}$ and see that $y:=X+\left(X^{2}-\right.$ $u p, p X)$ satisfies $C_{2}=A[y]=A+A y$, with $y^{2}=u p$ and $p y=0$. Next, by reworking the proof of (b), one can show that each element of $C_{u}$ can be uniquely expressed as the sum of an element of $A$ and an element of the form $v y$ where $v \in \mathcal{S} \cup\{0\}$. It follows easily that $\left|C_{u}\right|=p^{3}$. Moreover, the set of nilpotent elements of $C_{u}$ is the ideal $Q:=M+A y=M+(\mathcal{S} \cup\{0\}) y$ of cardinality $p^{2}$. It follows that $Q$ is the unique maximal ideal of $C_{u} ; Q \cap A=M ; Q^{2}=M \subset Q$; and the canonical ring homomorphism $A / M \rightarrow C_{u} / Q$ is an isomorphism (since it is an injective map of fields both of which have cardinality $p$ ). In addition, since $C_{u}=A+A y$ and $M y=0$, we see that $M$ is an ideal of $C_{u}$. Then $C_{u} / M$ must be a two-dimensional vector space over $\mathbb{F}_{p}(\cong A / M)$ since $\left|C_{u} / M\right|=\left|C_{u}\right| /|M|=p^{2}$. We have verified all the criteria in [12, Theorem $4.2(\mathrm{c})]$, and so $A \subset C_{u}$ is a ramified extension.

Next, we show that there cannot exist an $A$-algebra isomorphism $\varphi: B_{a} \rightarrow C_{u}$. Suppose, on the contrary, that such $\varphi$ exists. View $B_{a}=A(+) \mathbb{F}_{p}=A[\gamma]$ with $\gamma:=(0,1)$. As $\gamma^{2}=0$, we see by applying $\varphi$ that $z:=\varphi(\gamma) \in C_{u}$ must satisfy $A[z]=C_{u}$ and $z^{2}=0$. Consequently, $z \in Q \backslash M$. Therefore, as noted in the preceding paragraph (and using its notation), $z=m+s y$ for some $m \in M$ and some $s \in \mathcal{S}$. (Note that $s \neq 0$ since $z \notin M$.) Then $0=z^{2}=(m+s y)^{2}=m^{2}+2 s(m y)+s^{2} y^{2}=$ $0+2 s \cdot 0+s^{2} u p=s^{2} u p \neq 0$ (since $s^{2} u$ is not divisible by $p)$, the desired contradiction. Hence, $B_{a}$ is not isomorphic to $C_{u}$.

Next, to show that $C_{u_{1}} \cong C_{u_{2}}$ whenever $u_{1}$ and $u_{2}$ are quadratic residues of $p$, it is enough to show that if $C:=C_{1}$ and $u$ is a quadratic residue of $p$, then $C_{u} \cong C$. It suffices to prove that there exists $z \in C$ such that $C=A[z], z^{2}=u p$, and $p z=0$ (for then $C=A[z] \cong C_{u}$ ). By hypothesis, there exists $a \in \mathcal{S}$ such that $a^{2} \equiv u(\bmod p)$. By the definition of $C_{1}$, there exists $y \in C$ such that $C=A[y], y^{2}=p$, and $p y=0$. Put $z:=a y$. Since $a$ is a unit of $A$, we have $A z=(A a) y=A y$, and so $A+A z=A+A y=C$. It follows that $A[z]=C$. It is easy to check that $p z=0$. Moreover, since $a^{2}-u=t p$ for some "integer" $t$ and $p^{2}=0$, we get $z^{2}=a^{2} y^{2}=(u+t p) p=u p+t \cdot 0=u p$, as required.
Next, we will show that $C_{u_{1}} \cong C_{u_{2}}$ whenever $u_{1}$ and $u_{2}$ each are quadratic nonresidues of $p$. By the well known multiplicative property of the Legendre symbols [18, Theorem 9.3(ii)], $u_{1} u_{2}$ is a quadratic residue of $p$. Pick $a \in \mathcal{S}$ such that $u_{1} u_{2} \equiv a^{2}(\bmod p)$. Let $b$ be the element of $\mathcal{S}$ such that $(a+p \mathbb{Z}) /\left(u_{2}+p \mathbb{Z}\right)=b+p \mathbb{Z} \in \mathbb{F}_{p}$. Then, working in $\mathbb{F}_{p}$, we have $\left(u_{1}+p \mathbb{Z}\right) /\left(u_{2}+p \mathbb{Z}\right)$

$$
=\frac{\left(a^{2}+p \mathbb{Z}\right)}{\left(u_{2}^{2}+p \mathbb{Z}\right)}=\left[\frac{(a+p \mathbb{Z})}{\left(u_{2}+p \mathbb{Z}\right)}\right]^{2}=(b+p \mathbb{Z})^{2},
$$

and so $u_{1} \equiv b^{2} u_{2}(\bmod p)$. Given $z$ such that $C_{u_{2}}=A[z]$, $z^{2}=u_{2} p$, and $p z=0$, put $y:=b z \in C_{u_{2}}$. As $b$ is a unit in $A$, we see as above that $A+A y=A+A z$, and so $A[y]=C_{u_{2}}$. It is easy to check that $p y=0$. Moreover, for some "integer" $t$,

$$
\begin{aligned}
y^{2} & =b^{2} z^{2}=\left(b^{2} u_{2}\right) p=\left(u_{1}+t p\right) p=u_{1} p+t \cdot 0 \\
& =u_{1} p
\end{aligned}
$$

and so $C_{u_{2}}=A[y] \cong C_{u_{1}}$, as required.

Finally, to complete the proof, it suffices to show that if $u$ is a quadratic nonresidue of $p$, then $C:=C_{1}$ is not isomorphic to $C_{u}$. Suppose, on the contrary, that there exists an isomorphism $\psi: C \rightarrow C_{u}$. By hypothesis, there exists $y \in C$ such that $C=A[y], y^{2}=p$, and $p y=0$; and there exists $z \in C_{u}$ such that $C_{u}=A[z], z^{2}=u p$, and $p z=0$. Put $y^{*}:=\psi(y)$. Since $A \subset A[y]=C$, we see by applying the isomorphism $\psi$ that $A \subset A\left[y^{*}\right]=C_{u}$. Therefore, by (b), $y^{*}=a+\lambda z$ for some (uniquely determined) $a \in A$ and $\lambda \in \mathcal{S}$. Since $y$ is nilpotent, so is $y^{*}$. But $z$ is also nilpotent. Thus, $a$ is nilpotent; that is, $a \in M$. Hence $a^{2}=0$. In addition, $a y^{*}=\psi(a y) \in \psi(M y)=\psi(0)=0$; that is, $a y^{*}=0$. Also, $\left(y^{*}\right)^{2}=\psi\left(y^{2}\right)=\psi(p)=p$.

Consider $y^{*}-a=\lambda z \neq 0$. Squaring both sides leads to the equality of $\lambda^{2} z^{2}=\lambda^{2} u p$ with

$$
\left(y^{*}\right)^{2}-2 a y^{*}+a^{2}=p-2 \cdot 0+0=p .
$$

Thus $p=\lambda^{2} u p$; that is, $p\left(1-\lambda^{2} u\right)=0$. Hence, $1-\lambda^{2} u \equiv$ $0(\bmod p)$, and so $\lambda^{2} u \equiv 1(\bmod p)$. As $\lambda^{2}$ is obviously a quadratic residue of $p$ and $u$ is assumed to be a quadratic nonresidue of $p$, it follows from the multiplicative property of the Legendre symbols [18, Theorem 9.3(ii)] that $\lambda^{2} u$ is a quadratic nonresidue of $p$. Therefore, so is 1 , the desired contradiction. The proof is complete.

The five rings featured in parts (e) and (f) of Proposition 12 were identified in [19] as being, up to isomorphism, the (commutative unital) rings of cardinality 8 and (when $p$ is an odd prime number) $p^{3}$. We provided full details in proving those parts of Proposition 12 for two reasons: [19] provided few details relevant to this context and, unfortunately, there were some errors in [19]. In his Mathematical Review of [19], Kruse [20] listed the correct number (up to isomorphism) of (not necessarily commutative or unital) rings of cardinality $p^{3}$ and reported that a correct list of isomorphism class representatives for these 
rings had been given in [21]. See also the later treatment in [22] and the discussion of other relevant literature in Mal'tsev's Mathematical Review [23] of [22].

Proposition 12(e) gives some evidence concerning the essentiality of the role of the parameters given in Proposition 12(a). Indeed, for the case $p^{\alpha}=4$, we found that $B_{1}$ featured $i=2=j$, with $u$ and $v$ being nonuniquely determined units of $A$, while $B_{2}$ featured $i=1, j=2$, and $u=1$, with $v$ a nonuniquely determined unit of $A$. Moreover, not every ordered pair $(i, j)$ that fit the (necessary) conditions in Proposition 12(a) actually led to a viable candidate in Proposition 12(e). For readers who may wish to see the extent to which the behavior in parts (e) and (f) may be exhibited when $\alpha \geq 3$, we would suggest, rather than continuing to do computations by hand with the generator-and-relations information from Proposition 12(a), the use of appropriate computer software. We will comment further on such studies in the final remark of this paper.

We can now state our main results involving rings $R$ of positive characteristic. If the prime ring of $R$ is (isomorphic to) $\mathbb{Z} / n \mathbb{Z}$ and $n$ has the prime-power factorization $n=$ $\prod_{i=1}^{k} p_{i}^{\alpha_{i}}$, then by the Chinese Remainder Theorem, the prime ring of $R$ is $\prod_{i=1}^{k} \mathbb{Z} / p_{i}^{\alpha_{i}} \mathbb{Z}$. Accordingly, $R$ can be expressed as $R=\prod_{i=1}^{k} E_{i}$, where each $E_{i}$ is a suitable ring extension of the corresponding $\mathbb{Z} / p_{i}^{\alpha_{i}} \mathbb{Z}$. The forays into classification in the next two theorems are built from this point of view, featuring a lack of specificity only when a chain of minimal ring extensions from some $\mathbb{Z} / p_{i}^{\alpha_{i}} \mathbb{Z}$ to its corresponding $E_{i}$ includes a ramified extension of $\mathbb{Z} / p_{i}^{\alpha_{i}} \mathbb{Z}$, where $\alpha_{i} \geq 2$. As usual, if $E$ is any ring, the direct product of $E$ with an empty direct product is being viewed as $(E \times\{0\} \cong) E$.

Theorem 13. Up to isomorphism, the rings $R$ of positive characteristic that have exactly one proper subring can be characterized as follows. The prime ring of $R$ is (isomorphic to) the direct product $\prod_{i=1}^{k} \mathbb{Z} / p_{i}^{\alpha_{i}} \mathbb{Z}$, where $p=p_{1}, p_{2}, \ldots, p_{k}$ are pairwise distinct prime numbers for some positive integer $k$ and $\alpha=\alpha_{1}, \alpha_{2}, \ldots, \alpha_{k}$ are positive integers (and possibly $\alpha_{i}=\alpha_{j}$ for some $i \neq j$ ). Then (up to isomorphism), $R$ is the direct product $E \times \prod_{i=2}^{k} \mathbb{Z} / p_{i}^{\alpha_{i}} \mathbb{Z}$, where $E$ is a ring satisfying (exactly) one of the following five conditions:

(a) $\left(\alpha=1\right.$ and) $E=\mathbb{F}_{p^{q}}$, where $q$ is a prime number (which is possibly equal to $p$ );

(b) $\left(\alpha=1\right.$ and) $E=\mathbb{F}_{p} \times \mathbb{F}_{p}$;

(c) $\left(\alpha=1\right.$ and) $E=\mathbb{F}_{p}[X] /\left(X^{2}\right)$;

(d) $\alpha \geq 2$ and $E=\mathbb{Z} / p^{\alpha} \mathbb{Z} \times \mathbb{F}_{p}$;

(e) $\alpha \geq 2$ and $\mathbb{Z} / p^{\alpha} \mathbb{Z} \subset R$ is a ramified extension.

Moreover, in (a)-(e), the parameters $p_{1}, p_{2}, \ldots, p_{k}$, $\alpha_{1}, \alpha_{2}, \ldots, \alpha_{k}$ are determined by $R$.

Proof. By the above comments, the rings $R$ in question are the direct products of the asserted form in which $E$ is a minimal ring extension of $\mathbb{Z} / p^{\alpha} \mathbb{Z}$. If $\alpha=1$, then [2, Lemme 1.2] provides the classification given by (a), (b), and (c). If $\alpha \geq 2$, then Propositions 7 and 8 give that the suitable rings
$E$ are either decomposed extensions of $\mathbb{Z} / p^{\alpha} \mathbb{Z}$ or ramified extensions of $\mathbb{Z} / p^{\alpha} \mathbb{Z}$. By Proposition 10 , the former are given (of course, up to isomorphism) in (d); and the latter are stipulated in (e). As for the "Moreover" assertion, note that when $R$ (being a finite, hence an Artinian, ring) is expressed as a direct product $\prod_{i=1}^{k} E_{i}$ of nonzero local rings $E_{i}$, there is exactly one index $i$ such that $E_{i}$ is not a prime ring. This fact determines the prime number that plays the role of $p_{1}$, and the uniqueness of the other parameters is now clear.

By Proposition 12(d), condition (e) of Theorem 13 is nonvacuous for any prime number $p$ and any integer $\alpha \geq 2$. In case $\alpha=2$ and $p$ is any prime number, parts (e) and (f) of Proposition 12 serve to complete the classification of the rings $R$ figuring in condition (e) of Theorem 13. It would be interesting to know if such a classification could be completed for arbitrary prime-powers $p^{\alpha}$ when $\alpha \geq 3$.

We pause to isolate a result of some independent interest. Lemma 14 will be used often in the proof of Theorem 15.

Lemma 14. Let $A$ and $B$ be rings, with $E$ a ring extension of $A$. Put $C:=A \times B$ and $D:=E \times B$. Let $M$ be an ideal of $A$ and put $\mathscr{M}:=M \times B$. Then, consider the following:

(a) $C \subseteq D$ is a minimal ring extension if and if $A \subseteq E$ is a minimal ring extension.

(b) $C \subseteq D$ is an integral extension if and only if $A \subseteq E$ is an integral extension.

(c) $M \in \operatorname{Max}(C)$ if and only if $M \in \operatorname{Max}(A)$.

(d) $C \subseteq D$ is an integral minimal ring extension with crucial maximal ideal $M$ if and only if $A \subseteq E$ is an integral minimal ring extension with crucial maximal ideal M. Moreover, when these equivalent conditions hold, $C \subseteq D$ and $A \subseteq E$ are the same type of integral minimal ring extension (i.e., to say, inert, decomposed, or ramified).

Proof. (a) This is evident since $[C, D]=\{R \times B \mid R \in[A, E]\}$.

(b) Let $e \in E$ and $b \in B$. Straightforward calculations show that $(e, b)$ is integral over $C$ if and only if $e$ is integral over $A$.

(c) This is evident since $C / \mathscr{M} \cong A / M$.

(d) Note that $\mathscr{M D}=\mathscr{M}$ if and only if $M E=M$. Assume that these equivalent conditions hold. In view of (a), (b), and (c), the assertion now follows by considering the commutative diagram formed by the inclusion maps $C / \mathscr{M} \hookrightarrow D / \mathscr{M}$ and $A / M \hookrightarrow E / M$ and the isomorphisms $C / \mathscr{M} \rightarrow A / M$ and $D / \mathscr{M} \rightarrow E / M$.

Theorem 15. Up to isomorphism, the rings $R$ of positive characteristic that have exactly two proper subrings can be characterized as follows. The prime ring of $R$ is (isomorphic to) the direct product $\prod_{i=1}^{k} \mathbb{Z} / p_{i}^{\alpha_{i}} \mathbb{Z}$, where $p=p_{1}, p_{2}, \ldots, p_{k}$ are pairwise distinct prime numbers for some positive integer $k$ and $\alpha=\alpha_{1}, \alpha_{2}, \ldots, \alpha_{k}$ are positive integers (and possibly $\alpha_{i}=\alpha_{j}$ for some $i \neq j$ ). Then (up to isomorphism), $R$ is the direct product $E \times \prod_{i=2}^{k} \mathbb{Z} / p_{i}^{\alpha_{i}} \mathbb{Z}$, where $E$ is a ring satisfying (exactly) one of the following five conditions: 
(a) $E=\mathbb{F}_{p^{q^{2}}}$, where $q$ is a prime number (which is possibly equal to $p$ );

(b) $E=\mathbb{F}_{p^{q}} \times \mathbb{F}_{p}$, where $q$ is a prime number (which is possibly equal to $p$ );

(c) $E=R_{1}$, the ring that was constructed (in terms of any given prime number $p$ ) in Proposition 11;

(d) $\alpha \geq 2$ and $E=\mathbb{Z} / p^{\alpha} \mathbb{Z} \times \mathbb{F}_{p^{q}}$, where $q$ is a prime number (which is possibly equal to $p$ );

(e) $\alpha \geq 2$ and there exists a ring $B$ such that both $\mathbb{Z} / p^{\alpha} \mathbb{Z} \subset B$ and $B \subset R$ are ramified extensions and $\left(\mathbb{Z} / p^{\alpha} \mathbb{Z}\right)[u]=R$ for all $u \in R \backslash B$.

Proof. Let $S$ be a prime ring that is expressed as a direct product as in the statement of this result. A ring extension, say $R$, of $S$ can be expressed (up to isomorphism) as $\prod_{i=1}^{k} D_{i}$, where $D_{i}$ is a ring extension of $\mathbb{Z} / p_{i}^{\alpha_{i}} \mathbb{Z}$ for each $i=1, \ldots, k$. If we wish to consider only rings $R$ such that $|[S, R]|=3$, then there cannot exist distinct indices $i$ and $j$ such that $\mathbb{Z} / p_{i}^{\alpha_{i}} \mathbb{Z} \subset$ $D_{i}$ and $\mathbb{Z} / p_{j}^{\alpha_{j}} \mathbb{Z} \subset D_{j}$. Indeed, if one assumes the contrary, with $\{i, j\}=\{1,2\}$ for simplicity of notation, then

$$
\begin{gathered}
V:=D_{1} \times \prod_{i=2}^{k} \mathbb{Z} / p_{i}^{\alpha_{i}} \mathbb{Z}, \\
W:=\mathbb{Z} / p^{\alpha} \mathbb{Z} \times D_{2} \times \prod_{i=3}^{k} \mathbb{Z} / p_{i}^{\alpha_{i}} \mathbb{Z}
\end{gathered}
$$

are unequal members of $[S, R] \backslash\{S, R\}$, whence $|[S, R]| \geq 4$. This proves the assertion that we need only consider candidates $R$ expressible in the form $E \times \prod_{i=2}^{k} \mathbb{Z} / p_{i}^{\alpha_{i}} \mathbb{Z}$ as in the statement of this result. Therefore, by Lemma 14, our task translates to determining (up to isomorphism) those rings $E$ with prime ring $\mathbb{Z} / p^{\alpha} \mathbb{Z}$ such that $E$ has exactly two proper subrings. Building on Theorem 13, we will find many candidates. Those exhibiting the desired behavior will be deemed "good" (they will form the five mutually exclusive collections in the statement of this result), while the candidates that do not exhibit the desired behavior will be deemed "bad." The decision as to whether a given candidate is "good" or "bad" will be based on the conclusion in Theorem 2 concerning the condition from Theorem 1 that is satisfied by a tower of minimal ring extensions leading from $\mathbb{Z} / p^{\alpha} \mathbb{Z}$ to $E$. Any candidate for which Theorem 2(c) is applicable will be deemed "ambiguous." By Proposition 7, conditions (v) and (xiii) are the only conditions from Theorem 1 that can lead to the "ambiguous" designation. The candidates will be developed by taking as the "first step" of any such tower one of the rings identified in Theorem 13.

Our first candidates for suitable $E$ will come by building towers whose first step (from the prime ring $\mathscr{P}=\mathbb{F}_{p}$ ) arrives at the ring identified in Theorem 13(a), namely, $B=\mathbb{F}_{p^{q}}$, where $q$ is a prime number (which is possibly equal to $p$ ). For these candidates, the extension $B \subset E$ may be inert, decomposed, or ramified. The resulting towers $\mathscr{P} \subset B \subset$ $E$, respectively, satisfy conditions (v), (viii), and (xii) from Theorem 1. According to Theorem 2, these candidates are, respectively, ambiguous, bad, and bad. By [2, Lemme 1.2], that ambiguous candidate is $E=\mathbb{F}_{p^{q r}}$, where $r$ is a prime number (which is possibly equal to $p$ or $q$ ). The scrutiny of this candidate reveals two subcases. Indeed, if $q=r$, it follows from the classical Galois theory of finite fields that $B$ is the only ring (field, in this case) that is properly contained between $\mathbb{F}_{p}$ and $\mathbb{F}_{p^{q}}$. However, if $q \neq r$, then $\mathbb{F}_{p^{q}}$ and $\mathbb{F}_{p^{r}}$ are distinct fields that are properly contained between $\mathbb{F}_{p}$ and $\mathbb{F}_{p^{q r}}$. Accordingly, candidates from the first subcase are good (and this fact is recorded in part (a) of the statement of this result), while candidates from the second subcase are bad.

The next candidates for suitable $E$ will come via Theorem 13(b), building on the prime ring $\mathscr{P}=\mathbb{F}_{p}$ and the intermediate ring $B=\mathbb{F}_{p} \times \mathbb{F}_{p}$. For these candidates, the extension $B \subset E$ may be inert, decomposed, or ramified. The resulting towers $\mathscr{P} \subset B \subset E$, respectively, satisfy conditions (vi), (vii), and (x) from Theorem 1. According to Theorem 2, these candidates are, respectively, good, bad, and bad. By combining Lemma 14(d) with [2, Lemme 1.2], we find that good candidate to be (up to isomorphism) of the form $\mathbb{F}_{p^{q}} \times \mathbb{F}_{p}$, where $q$ is a prime number (which is possibly equal to $p$ ). This fact is recorded in part (b) of the statement of this result.

Next, we consider the candidates for $E$ coming via Theorem 13(c), building on the intermediate ring $B=$ $\mathbb{F}_{p}[X] /\left(X^{2}\right)$ (and its prime ring $\left.\mathscr{P}=\mathbb{F}_{p}\right)$. For these candidates, Proposition 8 shows that the extension $B \subset E$ may only be decomposed or ramified. The resulting towers $\mathscr{P} \subset$ $B \subset E$, respectively, satisfy conditions (ix) and (xiii) from Theorem 1. Accordingly, these candidates are, respectively, bad and ambiguous. In fact, an isomorphic copy of this bad candidate was already designated as "bad" in the preceding paragraph. As for the ambiguous candidate, there are two subcases. These were completely assessed in Proposition 11. The final assertion in the statement of that result produces part (c) of the statement of this result.

The next candidates for $E$ come via Theorem $13(\mathrm{~d})$, with $\mathscr{P}=\mathbb{Z} / p^{\alpha} \mathbb{Z}$, where $p$ is a prime number and the integer $\alpha \geq 2$, and $B=\mathbb{Z} / p^{\alpha} \mathbb{Z} \times \mathbb{F}_{p}$. By Lemma 14 and Proposition 8 , the relevant candidates $E$ are of two kinds: the "type 1 " candidates are those of the form $E_{1} \times \mathbb{F}_{p}$, where $\mathbb{Z} / p^{\alpha} \mathbb{Z} \subset E_{1}$ is either decomposed or ramified; the "type 2 " candidates are those of the form $\mathbb{Z} / p^{\alpha} \mathbb{Z} \times E_{2}$, where $\mathbb{F}_{p} \subset E_{2}$ is inert, decomposed, or ramified. By Lemma 14, the type 1 candidates, respectively, satisfy conditions (vii) and (x) from Theorem 1 and, hence, are deemed to be, respectively, bad and bad. The type 2 candidates, respectively, satisfy conditions (vi), (vii), and (x) from Theorem 1 and so are, respectively, good, bad, and bad. Thus (up to isomorphism), the sole good candidate emanating from Theorem $13(\mathrm{~d})$ is (thanks to [2, Lemme 1.2]) given by $\mathbb{Z} / p^{\alpha} \mathbb{Z} \times \mathbb{F}_{p q}$, where $q$ is a prime number (which is possibly equal to $p$ ). This fact is recorded in part (d) of the statement of this result.

Finally, we must address the candidates for $E$ that come via Theorem $13(\mathrm{e})$. These involve the prime ring $\mathscr{P}=\mathbb{Z} / p^{\alpha} \mathbb{Z}$, where $p$ is a prime number and the integer $\alpha \geq 2$. However, as we have classified the ramified extensions of the form $\mathbb{Z} / p^{\alpha} \mathbb{Z} \subset B$ only when $\alpha \leq 2$, we must settle for the formulation of part (e) of the statement of this result. 
Remark 16. (a) We next prove that condition (e) of Theorem 15 is nonvacuous for any $p^{\alpha}$ (where, as above, $p$ is a prime number and $\alpha$ is an integer exceeding 1$)$. Consider the tower $(A, M):=\mathbb{Z} / p^{\alpha} \mathbb{Z} \subset B:=A(+) M^{\alpha-1} \subset R:=$ $A(+) M^{\alpha-2}$. (As usual, $M^{0}:=A$.) Since

$$
M^{\alpha-1}=(p A)^{\alpha-1}=\frac{p^{\alpha-1} \mathbb{Z}}{p^{\alpha} \mathbb{Z}} \cong \frac{\mathbb{Z}}{p \mathbb{Z}} \cong \frac{A}{M},
$$

the usual combination of [15] and [16, Lemma 2.1] shows that $A \subset B$ is ramified (necessarily with maximal ideal $M$ ). Note that the unique prime ideal of $B$ is $N:=M(+) M^{\alpha-1}$. Identifying $A$ with $A(+) 0 \subset B$ as usual via $a \mapsto(a, 0)$ for each $a \in A$, we see that $N \cap A=M$. Next, to show that $B \subset R$ is ramified, it is straightforward to check the criteria given in [12, Theorem 4.2(c)]. (In detail, check that $(B: R)=N$; the unique prime ideal of $R$ is $Q:=M(+) M^{\alpha-2}$ and the canonical ring homomorphism $B / N \rightarrow R / Q$ is an isomorphism of fields; and $R / N$ is a two-dimensional vector space over $B / N\left(\cong \mathbb{F}_{p}\right)$, since as a vector space, $R / N \cong A / M \oplus$ $M^{\alpha-2} / M^{\alpha-1} \cong \mathbb{F}_{p} \oplus \mathbb{F}_{p} \cong B / N \oplus B / N$.) Of course, $Q \cap B=N$. Next, to show that the tower $A \subset B \subset R$ satisfies condition (e) of Theorem 15 (so that $R$ is a ring of characteristic $p^{\alpha}$ that has only two proper subrings), it suffices to show that if $u \in R \backslash B$, then $A[u]$ contains at least one element of $B \backslash A$. It will suffice to show that $p u \in B \backslash A$ (where $p u$ denotes the sum $u+\cdots+u$ of $p$ summands each of which is $u)$. We can write $u=\left(a, a^{*}\right)$ where $a \in A$ and $a^{*} \in M^{\alpha-2} \backslash M^{\alpha-1}=p^{\alpha-2} A \backslash p^{\alpha-1} A$. It remains only to observe that $p u=\left(p a, p a^{*}\right) \in B \backslash A$, the critical point being that $p a^{*} \neq 0 \in A$ since $a^{*} \notin p^{\alpha-1} A$.

(b) Not only is condition (e) of Theorem 15 nonvacuous (as we just proved in (a)), this condition is also not redundant. More precisely, one cannot delete the requirement " $\left(\mathbb{Z} / p^{\alpha} \mathbb{Z}\right)[u]=R$ for all $u \in R \backslash B$ " in the statement of condition (e) of Theorem 15. This can be shown for any $p^{\alpha}$ (where, as above, $p$ is a prime number and $\alpha$ is an integer exceeding 1$)$ as follows. As above, take $(A, M):=\mathbb{Z} / p^{\alpha} \mathbb{Z}$. Next, take $B:=A(+) \mathbb{F}_{p}$ (recalling that $A / M \cong \mathbb{F}_{p}$ ). Observe that the only prime ideal of $B$ is $N:=M(+) \mathbb{F}_{p} ; N \cap A=M$; and the canonical ring homomorphism $A / M \rightarrow B / N$ is an isomorphism of fields. Put $R:=B(+) \mathbb{F}_{p}(\cong B(+) B / N)$. Then the usual combination of [15] and [16, Lemma 2.1] shows that $A \subset B$ and $B \subset R$ are ramified extensions, with crucial maximal ideals $M$ and $N$, respectively. Of course, $R$ has only finitely many subrings because $R$ is finite. However, as we have seen in analyzing the rings called $D$ in the proofs of Theorem 6(2) and Proposition 11, such iterated idealizations always produce at least three proper subrings. For the present data, we have $A(+) 0(+) \mathbb{F}_{p} \in[A, R] \backslash\{A, B, R\}$.

(c) It may be useful to note that condition (e) of Theorem 15 is equivalent to the following condition, $(\mathrm{e})^{\prime}: \alpha \geq$ $2,|R|=p^{\alpha+2}, \operatorname{char}(R)=p^{\alpha}, R$ is subintegral over its prime ring, and there exists a proper subring $B$ of $R$ such that each element of $R \backslash B$ generates $R$ as a (unital) ring. By reasoning as in the second proof of Proposition 12(c), it is easy to show that $(\mathrm{e}) \Rightarrow(\mathrm{e})^{\prime}$. Conversely, assume $(\mathrm{e})^{\prime}$. Then there is no harm in viewing $A:=\mathbb{Z} / p^{\alpha} \mathbb{Z} \subset R$. To see that this is not a minimal ring extension, combine Propositions 7 and 8 with
Proposition $12(\mathrm{c})$. This leads to a $\operatorname{ring} B \in[A, R]$ such that $A \subset B$ is ramified, and then (e) follows easily.

The above formulation of (e) ${ }^{\prime}$ makes it clear that further progress on this program will depend on knowing the structure of certain local rings of prime-power cardinality $p^{n}$ when $n \geq 4$. According to the authors of [24], "All the commutative rings (of cardinality $p^{n}$ ) are known" because Wilson [25] has characterized the local ones. In our opinion, that characterization is somewhat noncommutative in spirit, inasmuch as it features matrix rings (over Galois rings). In a subsequent work, we hope to make that work sufficiently explicit so as to convert condition (e) of Theorem 15 into an explicit list that would be in the same spirit as the statements of the other conditions in Theorem 15.

(d) Despite the information in (a) and (b), one may wish for a fuller implementation of condition (e) of Theorem 15 that would require the classification (up to isomorphism) of all the ramified extensions of $\mathbb{Z} / p^{\alpha} \mathbb{Z}$ for all integers $\alpha \geq 2$ (and all prime numbers $p$ ). This was done only for $\alpha=2$ in Proposition 12 (e), (f). To investigate whether the behavior that was established there extends to the case $\alpha \geq 3$, we can suggest (in addition to pursuing the matrical methods of Wilson that were mentioned in (c)) a computer-aided study of the kind that was recommended following the proof of Proposition 12.

(e) Let $R$ be a ring with prime ring $A$. The above analysis has benefitted from the fact that if $R$ has at most two proper subrings, then $[A, R]$ is linearly ordered by inclusion. Any study that would go beyond the present work by seeking to classify (or even to nontrivially characterize) those $R$ having three proper subrings must deal with the loss of such linear order. For instance, it follows from [9, Lemma V.2] (and the second paragraph of the proof of Theorem 6) that the integral domains $C$ such that $\operatorname{char}(C)=0$ and $C$ has exactly three proper subrings are characterized, up to isomorphism, as the rings having the form $C=\mathbb{Z}[1 /(p q)]$, where $p$ and $q$ are distinct prime numbers. As we showed in the abovementioned proof, $[\mathbb{Z}, C]$ is not linearly ordered.

The above-noted increased level of complexity continues for positive characteristic. In the spirit of condition (a) in Theorem 15 (see also the second paragraph of [6, Remark 3.4(b)]), we will illustrate this by using field-theoretic examples. Let $p$ be a prime number. Then (up to isomorphism), the finite fields of characteristic $p$ that have exactly three proper subrings (subfields) are the fields having one of the forms $k_{1}=\mathbb{F}_{p^{q^{3}}}$ and $k_{2}=\mathbb{F}_{p^{q r}}$ where $q$ and $r$ are distinct prime numbers (one of which may be $p$ ). (The smallest such fields are $k_{1}=\mathbb{F}_{256}$ and $k_{2}=\mathbb{F}_{64}$.) In general, we have that $\left[\mathbb{F}_{p}, k_{1}\right]$ is linearly ordered and $\left[\mathbb{F}_{p}, k_{2}\right]$ is not linearly ordered.

The ring $R_{1}$ in the proof of Theorem 6(2) is isomorphic to the 2-trivial extension $\mathbb{Z} \ltimes_{2} \mathbb{F}_{p} z \ltimes \mathbb{F}_{p} x$ (in the sense of [26]). The theory developed in [26] can illuminate such constructions. For instance, the assertion that $N_{1}$ is a prime ideal of $R_{1}$ in the above-mentioned proof can be seen via [26, Theorem 4.7].

\section{Competing Interests}

The author declares that there is no conflict of interests in the publication of this paper. 


\section{References}

[1] D. E. Dobbs, "When the juxtaposition of two minimal ring extensions produces no new intermediate rings," Palestine Journal of Mathematics, vol. 6, no. 1, 2017.

[2] D. Ferrand and J.-P. Olivier, "Homomorphismes minimaux d'anneaux," Journal of Algebra, vol. 16, pp. 461-471, 1970.

[3] D. E. Dobbs and J. Shapiro, "A classification of the minimal ring extensions of an integral domain," Journal of Algebra, vol. 305, no. 1, pp. 185-193, 2006.

[4] D. D. Anderson, D. E. Dobbs, and B. Mullins, "The primitive element theorem for commutative algebras," Houston Journal of Mathematics, vol. 25, no. 4, pp. 603-623, 1999.

[5] I. Kaplansky, Commutative Rings, University of Chicago Press, Chicago, Ill, USA, 1974.

[6] D. E. Dobbs and J. Shapiro, "When only finitely many intermediate rings result from juxtaposing two minimal ring extensions," Palestine Journal of Mathematics, vol. 5, pp. 13-31, 2016.

[7] D. E. Dobbs, G. Picavet, and M. Picavet-L'Hermitte, "Characterizing the ring extensions that satisfy FIP or FCP," Journal of Algebra, vol. 371, pp. 391-429, 2012.

[8] J. A. Huckaba, Commutative rings with zero divisors, vol. 117 of Monographs and Textbooks in Pure and Applied Mathematics, Marcel Dekker, New York, NY, USA, 1988.

[9] D. E. Dobbs, B. Mullins, G. Picavet, and M. Picavet-L'Hermitte, "On the FIP property for extensions of commutative rings," Communications in Algebra, vol. 33, no. 9, pp. 3091-3119, 2005.

[10] M. S. Gilbert, Extensions of commutative rings with linearly ordered intermediate rings [Ph.D. dissertation], University of Tennessee, Knoxville, Tenn, USA, 1996.

[11] D. Lazard, "Autour de la platitude," Bulletin de la Société Mathématique de France, vol. 97, pp. 81-128, 1969.

[12] D. E. Dobbs, G. Picavet, M. Picavet-L'Hermitte, and J. Shapiro, "On intersections and composites of minimal ring extensions," JP Journal of Algebra, Number Theory and Applications, vol. 26, no. 2, pp. 103-158, 2012.

[13] D. E. Dobbs and J. Shapiro, "A classification of the minimal ring extensions of certain commutative rings," Journal of Algebra, vol. 308, no. 2, pp. 800-821, 2007.

[14] D. E. Dobbs, B. Mullins, and M. Picavet-L'Hermitte, "The singly generated unital rings with only finitely many unital subrings," Communications in Algebra, vol. 36, no. 7, pp. 2638-2653, 2008.

[15] D. E. Dobbs, "Every commutative ring has a minimal ring extension," Communications in Algebra, vol. 34, no. 10, pp. 38753881, 2006.

[16] G. Picavet and M. Picavet-L'Hermitte, "Modules with finitely many submodules," International Electronic Journal of Algebra, vol. 19, pp. 119-131, 2016.

[17] O. Zariski and P. Samuel, Commutative algebra, Volume I, The University Series in Higher Mathematics, D. Van Nostrand, Princeton, NJ, USA, 1958.

[18] K. H. Rosen, Elementary Number Theory and its Applications, Addison-Wesley, Reading, Mass, USA, 3rd edition, 1993.

[19] R. Gilmer and J. Mott, "Associative rings of order $P^{3}$," Proceedings of the Japan Academy, vol. 49, pp. 795-799, 1973.

[20] R. L. Kruse, Article ID 0369422, MR 0369422 (51 \# 5655), Mathematical Reviews Division of the American Mathematical Society, Providence, 1976.

[21] R. Ballieu, "Anneaux finis; systèmes hypercomplexes de rang trois sur un corps commutatif," Annales de la Société Scientifique de Bruxelles. Série I, vol. 61, pp. 222-227, 1947.
[22] V. G. Antipkin and V. P. Elizarov, "Rings of order $p^{3}$," Sibirskii Matematicheskii Zhurnal, vol. 23, no. 4, pp. 9-18, 1982.

[23] Y. N. Mal'tsev, Mathematical Reviews, American Mathematical Society, Providence, RI, USA, 1984.

[24] J. B. Derr, G. F. Orr, and P. S. Peck, "Noncommutative rings of order $p^{4}$," Journal of Pure and Applied Algebra, vol. 97, no. 2, pp. 109-116, 1994.

[25] R. S. Wilson, "Representations of finite rings," Pacific Journal of Mathematics, vol. 53, pp. 647-649, 1974.

[26] D. D. Anderson, D. Bennis, B. Fahid, and A. Shaiea, "n-trivial extensions of rings," https://arxiv.org/abs/1604.01486. 


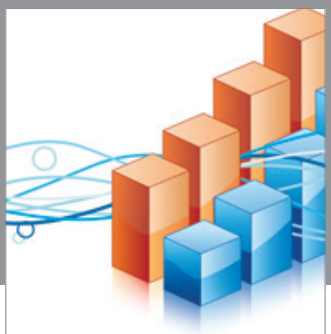

Advances in

Operations Research

vatem alat4

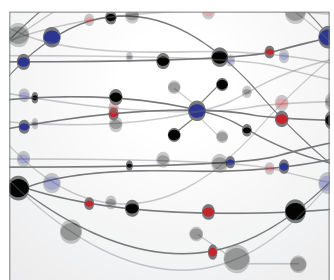

\section{The Scientific} World Journal
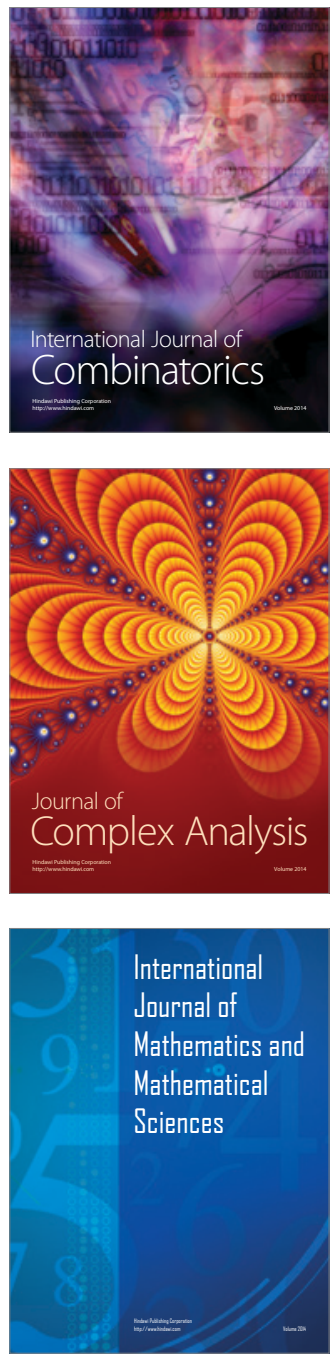
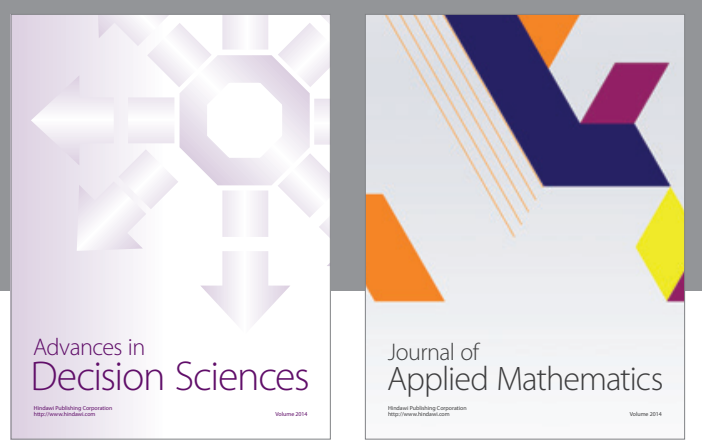

Algebra

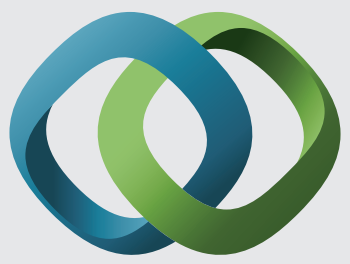

\section{Hindawi}

Submit your manuscripts at

http://www.hindawi.com
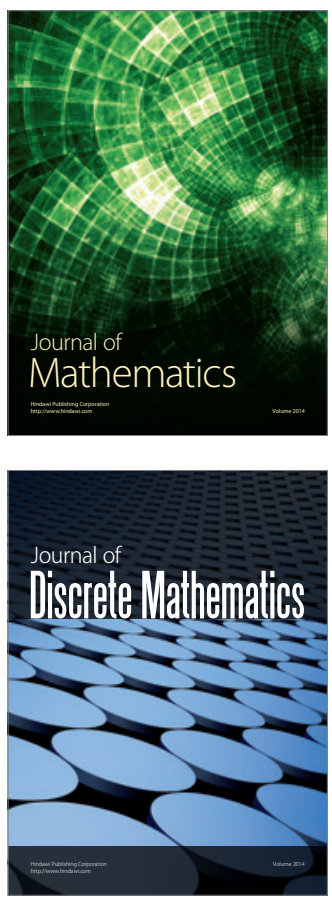

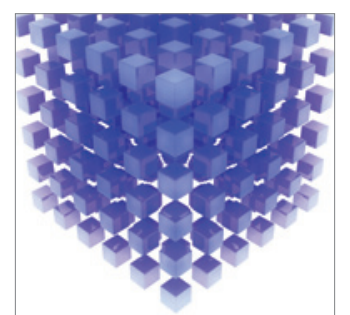

Mathematical Problems in Engineering
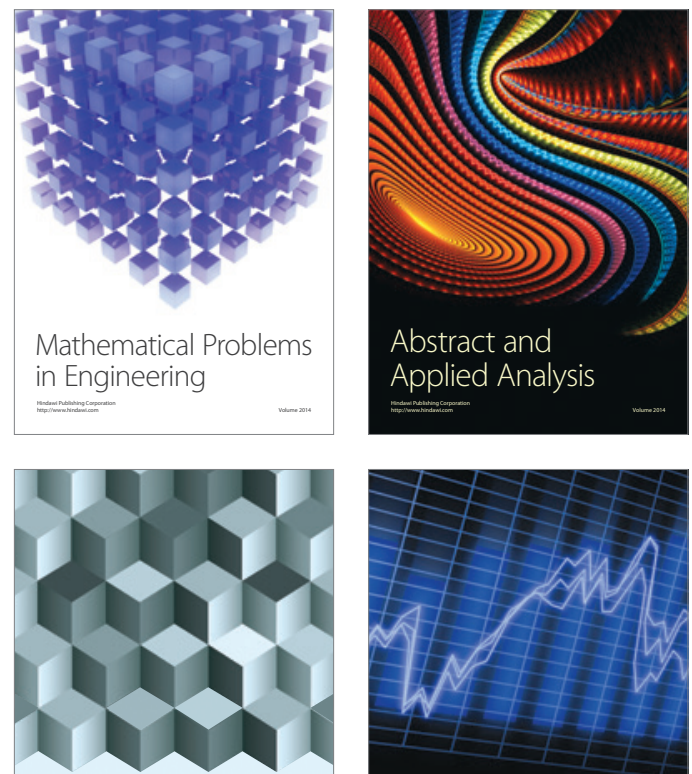

Journal of

Function Spaces

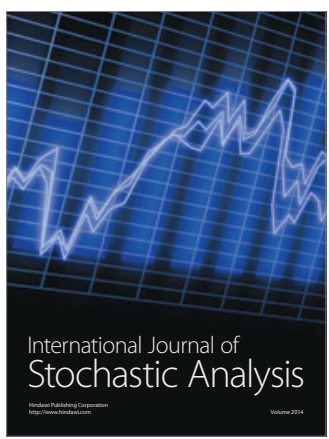

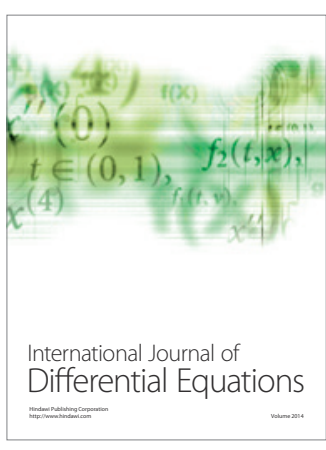
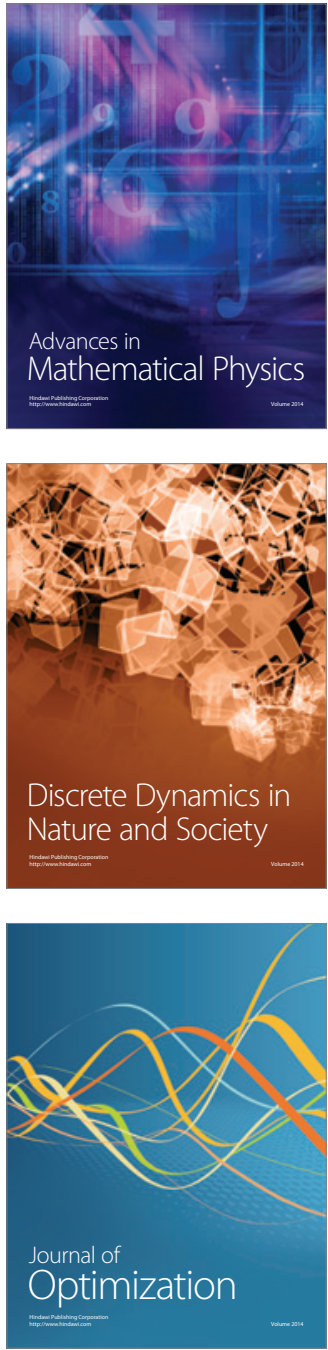